\title{
Substituent Effects in Eight-Electron Electrocyclic Reactions
}

\author{
Begoña Lecea, ${ }^{a}$ Ana Arrieta ${ }^{b}$ and Fernando P. Cossío ${ }^{b, *}$ \\ Farmazi Fakultatea and Kimika Fakultatea \\ Universidad del País Vasco-Euskal Herriko Unibertsitatea, \\ P.K. 1072, 20080 San Sebastián-Donostia, Spain \\ qopcomof@sq.ehu.es
}

Supporting Information

\section{List of Contents}

\begin{tabular}{|l|l|}
\hline Content & Page \\
\hline $\begin{array}{l}\text { Energies (including zero-point vibrational energies, ZPVE) and number of } \\
\text { imaginary frequencies (NIMAG) of all the stationary points discussed in the } \\
\text { paper. }\end{array}$ & \\
\hline Cartesian coordinates (in A) of all the stationary points discussed in the paper. & $\mathrm{S} 5$ \\
\hline $\begin{array}{l}\text { Figure S1. Chief geometric features (B3LYP/6-31G* level of theory) of } \\
\text { transition structures in- and } \text { out-TSb-g. }\end{array}$ & $\mathrm{S} 43$ \\
\hline $\begin{array}{l}\text { Figure S2. Chief geometric features (B3LYP/6-31G* level of theory) of } \\
\text { transition structures in- and } \text { out-TSh-I. }\end{array}$ & $\mathrm{S} 44$ \\
\hline
\end{tabular}

\footnotetext{
${ }^{a}$ Farmazi Fakultatea.

${ }^{\mathrm{b}}$ Kimika Fakultatea.
} 
Energies (including zero-point vibrational energies, ZPVE) and number of imaginary frequencies (NIMAG) of all the stationary points discussed in the paper.

\begin{tabular}{|c|c|c|c|}
\hline & NIMAG & Energy (a.u.) & ZPVE (a.u.) \\
\hline TS1a & 1 & -155.91655 & 0.08422 \\
\hline out-TS1b & 1 & -211.28276 & 0.10185 \\
\hline in-TS1b & 1 & -211.25896 & 0.10139 \\
\hline out-TS1c & 1 & -255.15807 & 0.07707 \\
\hline in-TS1c & 1 & -255.13373 & 0.07638 \\
\hline out-TS1d & 1 & -615.52331 & 0.07544 \\
\hline in-TS1d & 1 & -615.50004 & 0.07544 \\
\hline out-TS1e & 1 & -2727.03115 & 0.07475 \\
\hline in-TS1e & 1 & -2727.00978 & 0.07443 \\
\hline out-TS1f & 1 & -195.23663 & 0.11210 \\
\hline in-TS1f & 1 & -195.22722 & 0.11256 \\
\hline out-TS1g & 1 & -211.63127 & 0.11594 \\
\hline in-TS1g & 1 & -211.62170 & 0.11686 \\
\hline out-TS1h & 1 & -492.95630 & 0.08970 \\
\hline in-TS1h & 1 & -492.95256 & 0.08975 \\
\hline out-TS1i & 1 & -248.16460 & 0.08336 \\
\hline in-TS1i & 1 & -248.15734 & 0.08323 \\
\hline out-TS1j & 1 & -360.42355 & 0.08756 \\
\hline in-TS $1 \mathrm{j}$ & 1 & -360.41390 & 0.08744 \\
\hline out-TS1k & 1 & -269.24144 & 0.09398 \\
\hline in-TS1k & 1 & -269.24771 & 0.09443 \\
\hline out-TS11 & 1 & -285.21144 & 0.08103 \\
\hline in-TS11 & 1 & -285.21777 & 0.08165 \\
\hline $3 a$ & 0 & -310.79927 & 0.15328 \\
\hline (E) $3 \mathrm{~b}$ & 0 & -366.15671 & 0.17068 \\
\hline (Z) $3 \mathrm{~b}$ & 0 & -366.15471 & 0.17067 \\
\hline (E) $3 \mathrm{c}$ & 0 & -410.03027 & 0.14582 \\
\hline (Z) $3 \mathrm{c}$ & 0 & -410.03132 & 0.14600 \\
\hline (E) $3 \mathrm{~d}$ & 0 & -770.39691 & 0.14429 \\
\hline (Z) $3 d$ & 0 & -770.39685 & 0.14446 \\
\hline (E) $3 \mathrm{e}$ & 0 & -2881.90445 & 0.14372 \\
\hline (Z) $3 \mathrm{e}$ & 0 & -2881.90511 & 0.14391 \\
\hline (E) $3 \mathrm{f}$ & 0 & -350.11959 & 0.18149 \\
\hline (Z) $3 \mathrm{f}$ & 0 & -350.11692 & 0.18159 \\
\hline (E) $3 \mathrm{~g}$ & 0 & -366.51223 & 0.18512 \\
\hline (Z) $3 \mathrm{~g}$ & 0 & -366.51218 & 0.18504 \\
\hline (E) $3 \mathrm{~h}$ & 0 & -647.83905 & 0.15881 \\
\hline (Z) $3 \mathrm{~h}$ & 0 & -647.83479 & 0.15902 \\
\hline$(E) 3 \mathrm{i}$ & 0 & -403.04680 & 0.15242 \\
\hline (Z) $3 \mathrm{i}$ & 0 & -403.04630 & 0.15260 \\
\hline (E) $3 \mathrm{j}$ & 0 & -515.30383 & 0.15665 \\
\hline (Z) $3 \mathrm{j}$ & 0 & -515.30004 & 0.15680 \\
\hline
\end{tabular}




\begin{tabular}{|c|c|c|c|}
\hline (E) $3 \mathrm{k}$ & 0 & -424.12728 & 0.16319 \\
\hline (Z) $3 \mathrm{k}$ & 0 & -424.12219 & 0.16322 \\
\hline (E) 31 & 0 & -440.09732 & 0.15077 \\
\hline (Z) 31 & 0 & -440.09510 & 0.15092 \\
\hline$(Z, Z, Z, Z) 3 \mathrm{~m}$ & 0 & -389.43453 & 0.21001 \\
\hline$(E, Z, Z, E) 3 \mathrm{~m}$ & 0 & -389.43981 & 0.20970 \\
\hline$(E, Z, Z, Z) 3 \mathrm{~m}$ & 0 & -389.43720 & 0.21002 \\
\hline $\mathrm{TSa}$ & 1 & -310.77367 & 0.15449 \\
\hline TS'a & 1 & -310.72751 & 0.15201 \\
\hline out-TSb & 1 & -366.12581 & 0.17162 \\
\hline in-TSb & 1 & -366.12870 & 0.17252 \\
\hline out-TSc & 1 & -410.00497 & 0.14682 \\
\hline in-TSc & 1 & -410.00281 & 0.14687 \\
\hline out-TSd & 1 & -770.36986 & 0.14542 \\
\hline in-TSd & 1 & -770.36103 & 0.14526 \\
\hline out-TSe & 1 & -2881.87860 & 0.14471 \\
\hline in-TSe & 1 & -2881.87098 & 0.14443 \\
\hline out-TSf & 1 & -350.09184 & 0.18272 \\
\hline in-TSf & 1 & -350.08564 & 0.18277 \\
\hline out-TSg & 1 & -366.48589 & 0.18646 \\
\hline in-TSg & 1 & -366.49470 & 0.18636 \\
\hline out-TSh & 1 & -647.81319 & 0.15979 \\
\hline in-TSh & 1 & -647.80061 & 0.15950 \\
\hline out-TSi & 1 & -403.01941 & 0.15362 \\
\hline in-TSi & 1 & -403.01307 & 0.15339 \\
\hline out-TSj & 1 & -515.27744 & 0.15765 \\
\hline in-TSj & 1 & -515.26843 & 0.15747 \\
\hline out-TSk & 1 & -424.09913 & 0.16431 \\
\hline in-TSk & 1 & -424.09171 & 0.16409 \\
\hline out-TS1 & 1 & -440.06853 & 0.15182 \\
\hline in-TSl & 1 & -440.06084 & 0.15135 \\
\hline in, in-TSm & 1 & -389.39670 & 0.21114 \\
\hline out, out-TSm & 1 & -389.40967 & 0.21109 \\
\hline out, in-TSm & 1 & -389.40310 & 0.21094 \\
\hline $4 a$ & 0 & -310.81184 & 0.15748 \\
\hline $4 b$ & 0 & -366.15466 & 0.17481 \\
\hline $4 c$ & 0 & -410.04411 & 0.14947 \\
\hline $4 d$ & 0 & -770.40895 & 0.14812 \\
\hline $4 e$ & 0 & -2881.91925 & 0.14749 \\
\hline $4 \mathrm{f}$ & 0 & -350.12587 & 0.18568 \\
\hline $4 g$ & 0 & -366.53268 & 0.18985 \\
\hline $4 h$ & 0 & -647.85101 & 0.16259 \\
\hline $4 i$ & 0 & -403.04867 & 0.15594 \\
\hline $4 j$ & 0 & -515.31153 & 0.16022 \\
\hline $4 \mathrm{k}$ & 0 & -424.12599 & 0.16620 \\
\hline 41 & 0 & -440.09019 & 0.153 \\
\hline
\end{tabular}


trans $-4 \mathrm{~m}$

cis $-4 \mathrm{~m}$

Ts2a

TS3a

TS4a
0

0

1

1

1
$-389.43736$

$-389.43171$

$-310.74205$

$-310.71693$

$-310.71887$
0.21382

0.21409

0.15252

0.15088

0.15126 
Cartesian coordinates (in $\AA$ ) of all the stationary points discussed in the paper.

TS1a

\begin{tabular}{|c|c|c|c|c|}
\hline \multirow{2}{*}{$\begin{array}{l}\text { Atomic } \\
\text { Number }\end{array}$} & Coordinate & \multicolumn{2}{|c|}{ (Angstroms) } & \multirow[b]{2}{*}{$\mathrm{Z}$} \\
\hline & $\mathrm{X}$ & $\mathrm{Y}$ & & \\
\hline 6 & -1.065231 & -0.625647 & 0.119127 & \\
\hline 6 & 1.065232 & -0.625646 & -0.119127 & \\
\hline 6 & 0.683368 & 0.733029 & 0.083900 & \\
\hline 6 & -0.683368 & 0.733029 & -0.083901 & \\
\hline 1 & -1.882977 & -1.099942 & -0.434905 & \\
\hline 1 & -0.871488 & -1.085861 & 1.080725 & \\
\hline 1 & 0.871489 & -1.085863 & -1.080724 & \\
\hline 1 & 1.882978 & -1.099940 & 0.434907 & \\
\hline 1 & 1.339533 & 1.541509 & 0.403350 & \\
\hline 1 & -1.339534 & 1.541509 & -0.403348 & \\
\hline
\end{tabular}

\section{out-TS1b}

\begin{tabular}{|c|c|c|c|c|}
\hline \multirow{2}{*}{$\begin{array}{l}\text { Atomic } \\
\text { Number }\end{array}$} & \multicolumn{4}{|c|}{ Coordinates (Angstroms) } \\
\hline & $\mathrm{X}$ & $\mathrm{Y}$ & \multicolumn{2}{|r|}{$\mathrm{z}$} \\
\hline 6 & -0.646435 & -0.025944 & 0.385114 & \\
\hline 6 & 1.186650 & -1.002217 & -0.116211 & \\
\hline 7 & -1.932938 & -0.118223 & -0.127059 & \\
\hline 6 & 1.479660 & 0.398327 & -0.052978 & \\
\hline 6 & 0.260387 & 1.022581 & -0.008721 & \\
\hline 1 & -0.557362 & -0.427234 & 1.387928 & \\
\hline 1 & 0.695739 & -1.382156 & -1.006639 & \\
\hline 1 & 1.819466 & -1.749219 & 0.374904 & \\
\hline 1 & -2.028579 & 0.004188 & -1.127241 & \\
\hline 1 & -2.515957 & -0.864900 & 0.227871 & \\
\hline 1 & 2.464858 & 0.851794 & 0.063423 & \\
\hline 1 & -0.029167 & 2.038605 & -0.274051 & \\
\hline \multicolumn{5}{|l|}{ in-TS1b } \\
\hline \multicolumn{5}{|c|}{ Coordinates (Angstroms) } \\
\hline Number & $\mathrm{X}$ & $\mathrm{Y}$ & & $\mathrm{Z}$ \\
\hline \multicolumn{5}{|c|}{ 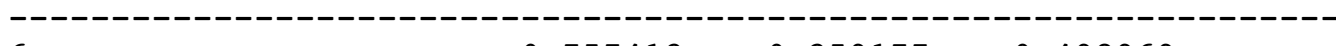 } \\
\hline 6 & 0.757413 & 0.259177 & 0.498969 & \\
\hline 6 & -1.069575 & -1.016371 & 0.256721 & \\
\hline 7 & 1.699602 & -0.267884 & -0.397390 & \\
\hline 6 & -1.307262 & 0.230324 & -0.394866 & \\
\hline 6 & -0.334105 & 1.094259 & 0.045553 & \\
\hline 1 & 1.232563 & 0.440208 & 1.472988 & \\
\hline 1 & -1.083223 & -1.039373 & 1.340503 & \\
\hline 1 & -1.297224 & -1.980693 & -0.208654 & \\
\hline 1 & 1.295180 & -0.460263 & -1.310487 & \\
\hline 1 & 2.177601 & -1.093347 & -0.044990 & \\
\hline 1 & -2.104097 & 0.433724 & -1.114984 & \\
\hline 1 & -0.396836 & 2.170600 & 0.209092 & \\
\hline \multicolumn{5}{|l|}{ out-TS1C } \\
\hline Atomic & Coordinat & s (Angstroms & & \\
\hline Number & $\mathrm{X}$ & $\mathrm{Y}$ & & $\mathrm{Z}$ \\
\hline
\end{tabular}




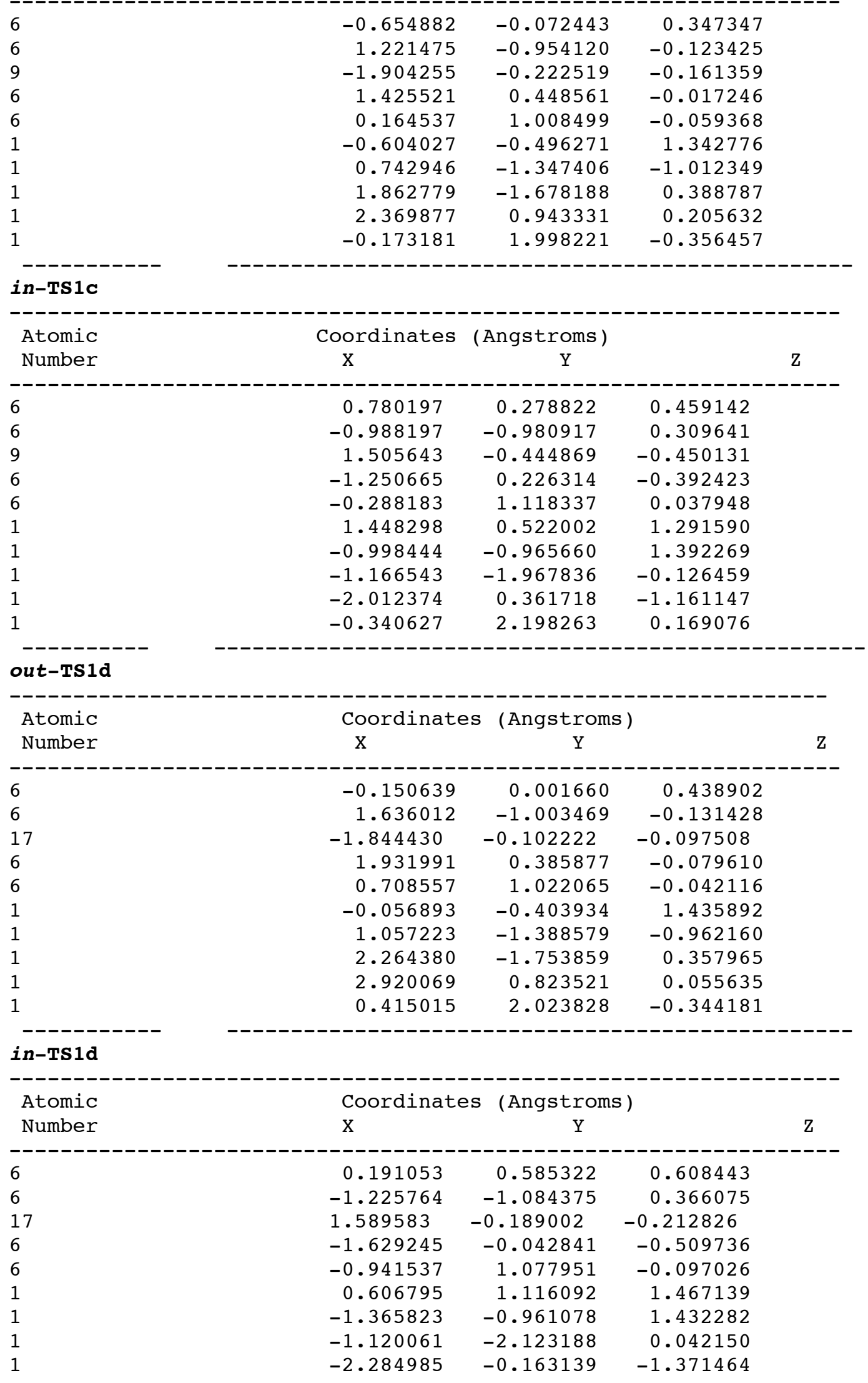




$\begin{array}{lrrr}6 & 0.682524 & 0.405638 & 0.502296 \\ 6 & -0.951988 & -1.041617 & 0.313238 \\ 6 & 1.682907 & -0.338593 & -0.362741 \\ 6 & -1.335714 & 0.118065 & -0.416258 \\ 6 & -0.459463 & 1.099878 & -0.010804 \\ 1 & 1.167363 & 0.810493 & 1.403998 \\ 1 & -0.960202 & -1.005404 & 1.396137 \\ 1 & -1.039704 & -2.054863 & -0.093411 \\ 1 & 1.231711 & -0.690167 & -1.293843 \\ 1 & 2.123113 & -1.195564 & 0.160551 \\ 1 & 2.516508 & 0.335356 & -0.619309 \\ 1 & -2.119893 & 0.165220 & -1.172215 \\ 1 & -0.628495 & 2.174710 & 0.063700\end{array}$

\section{out-TS1g}

\begin{tabular}{|c|c|c|c|c|}
\hline Atomic & \multicolumn{3}{|c|}{ Coordinates (Angstroms) } & \\
\hline Number & $X$ & $\mathrm{Y}$ & & $\mathrm{Z}$ \\
\hline 6 & -0.518728 & -0.097785 & 0.360542 & \\
\hline 6 & 1.376248 & -0.954030 & -0.132085 & \\
\hline 7 & -1.982075 & -0.177403 & -0.114588 & \\
\hline 6 & 1.539848 & 0.454439 & -0.026161 & \\
\hline 6 & 0.273050 & 0.998010 & -0.063156 & \\
\hline 1 & -0.483660 & -0.495903 & 1.366307 & \\
\hline 1 & 0.905821 & -1.366783 & -1.015541 & \\
\hline 1 & 2.019095 & -1.657469 & 0.402665 & \\
\hline 1 & -2.076411 & 0.142719 & -1.085326 & \\
\hline 1 & -2.617201 & 0.389894 & 0.466199 & \\
\hline 1 & -2.322903 & -1.145627 & -0.077590 & \\
\hline 1 & 2.474393 & 0.959305 & 0.205420 & \\
\hline 1 & -0.047115 & 2.011873 & -0.294857 & \\
\hline
\end{tabular}

\section{in-TS1g}

\begin{tabular}{|c|c|c|c|c|}
\hline Atomic & \multicolumn{3}{|c|}{ Coordinates (Angstroms) } & \\
\hline Number & $\mathrm{x}$ & $\mathrm{Y}$ & & Z \\
\hline \multicolumn{4}{|l|}{--ー-ー--ー } & \\
\hline 6 & 0.640098 & 0.412593 & 0.569383 & \\
\hline 6 & -1.006776 & -1.023598 & 0.333043 & \\
\hline 7 & 1.568840 & -0.351571 & -0.352187 & \\
\hline 6 & -1.314421 & 0.135545 & -0.441206 & \\
\hline 6 & -0.451243 & 1.121880 & -0.009209 & \\
\hline 1 & 1.254022 & 0.808440 & 1.379918 & \\
\hline 1 & -1.070946 & -0.956365 & 1.412114 & \\
\hline 1 & -1.120444 & -2.039965 & -0.057405 & \\
\hline 1 & 1.062140 & -0.659637 & -1.191184 & \\
\hline 1 & 1.969716 & -1.182533 & 0.100595 & \\
\hline 1 & 2.359122 & 0.234721 & -0.662995 & \\
\hline 1 & -2.058029 & 0.176848 & -1.234591 & \\
\hline 1 & -0.583402 & 2.200968 & 0.006781 & \\
\hline \multicolumn{5}{|c|}{ out-TS1h } \\
\hline Atomic & \multicolumn{3}{|c|}{ Coordinates (Angstroms) } & \\
\hline Number & $\mathrm{x}$ & $\mathrm{Y}$ & & $\mathrm{Z}$ \\
\hline 6 & -0.375218 & 0.033580 & -0.517529 & \\
\hline
\end{tabular}




$\begin{array}{rrrr}6 & -2.155280 & -1.008884 & 0.069223 \\ 6 & 1.044001 & -0.038960 & -0.028641 \\ 6 & -2.490564 & 0.375511 & 0.015891 \\ 6 & -1.282698 & 1.030162 & -0.044841 \\ 9 & 1.131003 & -0.676436 & 1.166152 \\ 9 & 1.568465 & 1.198125 & 0.154219 \\ 9 & 1.845306 & -0.701239 & -0.889760 \\ 1 & -0.521042 & -0.403811 & -1.497406 \\ 1 & -1.546141 & -1.370831 & 0.888252 \\ 1 & -2.760481 & -1.783961 & -0.411854 \\ 1 & -3.495987 & 0.783127 & -0.069624 \\ 1 & -1.020771 & 2.042963 & 0.250518\end{array}$

\section{in-TS1h}

\begin{tabular}{|c|c|c|c|}
\hline Atomic & \multicolumn{3}{|c|}{ Coordinates (Angstroms) } \\
\hline Number & $\mathrm{X}$ & $\mathrm{Y}$ & $\mathrm{Z}$ \\
\hline 6 & 0.274348 & 0.582176 & -0.765117 \\
\hline 6 & 1.635902 & -1.067630 & -0.307221 \\
\hline 6 & -0.876563 & 0.000092 & 0.012695 \\
\hline 6 & 2.097179 & 0.076284 & 0.399158 \\
\hline 6 & 1.429382 & 1.146661 & -0.153295 \\
\hline 9 & -0.587458 & -0.429551 & 1.253790 \\
\hline 9 & -1.460932 & -1.025963 & -0.653861 \\
\hline 9 & -1.841902 & 0.952917 & 0.150150 \\
\hline 1 & -0.127588 & 1.001937 & -1.694279 \\
\hline 1 & 1.753258 & -1.106838 & -1.382743 \\
\hline 1 & 1.524628 & -2.046285 & 0.167161 \\
\hline 1 & 2.770838 & 0.058169 & 1.254258 \\
\hline 1 & 1.730006 & 2.190889 & -0.212431 \\
\hline
\end{tabular}

out-TS1i

\begin{tabular}{|c|c|c|c|c|}
\hline Atomic & \multicolumn{4}{|c|}{ Coordinates (Angstroms) } \\
\hline Number & $\mathrm{X}$ & $\mathrm{Y}$ & & $\mathrm{Z}$ \\
\hline 6 & -0.247769 & 0.036544 & 0.490519 & \\
\hline 6 & 1.499224 & -1.036344 & -0.135738 & \\
\hline 6 & -1.619226 & -0.053932 & 0.086386 & \\
\hline 7 & -2.722218 & -0.156582 & -0.276083 & \\
\hline 6 & 1.850403 & 0.350245 & -0.147433 & \\
\hline 6 & 0.660545 & 1.019955 & -0.045058 & \\
\hline 1 & -0.051698 & -0.364146 & 1.478632 & \\
\hline 1 & 0.876713 & -1.428089 & -0.931116 & \\
\hline 1 & 2.109309 & -1.795343 & 0.363573 & \\
\hline 1 & 2.862715 & 0.749301 & -0.138896 & \\
\hline 1 & 0.399423 & 2.035545 & -0.331669 & \\
\hline
\end{tabular}

in-TS1i

$\begin{array}{lrrr}\text { Atomic } & \text { Coordinates } & \text { (Angstroms) } & \text { Z } \\ \text { Number } & X & Y & \\ -0.192024 & -0.705458 & 0.580112 \\ 6 & 0.974376 & 1.130282 & 0.418419 \\ 6 & -1.355927 & -0.089750 & 0.015014 \\ 6 & -2.347071 & 0.351314 & -0.412404\end{array}$




\begin{tabular}{rrrr}
6 & 1.515660 & 0.212847 & -0.533791 \\
6 & 0.976910 & -1.002858 & -0.211751 \\
1 & -0.445776 & -1.331801 & 1.442878 \\
1 & 1.171036 & 0.972637 & 1.471822 \\
1 & 0.692926 & 2.154605 & 0.161832 \\
1 & 2.153063 & 0.479759 & -1.374479 \\
1 & 1.344281 & -2.004776 & -0.423250 \\
\hline
\end{tabular}

out-TS $1 j$

\begin{tabular}{|c|c|c|c|c|}
\hline \multirow{2}{*}{$\begin{array}{l}\text { Atomic } \\
\text { Number }\end{array}$} & \multicolumn{4}{|c|}{ Coordinates (Angstroms) } \\
\hline & $\mathrm{x}$ & $\mathrm{Y}$ & & Z \\
\hline 6 & -0.094188 & -0.172797 & -0.436958 & \\
\hline 6 & -1.847516 & -0.942539 & 0.488228 & \\
\hline 7 & 1.301105 & -0.025642 & -0.012241 & \\
\hline 6 & -2.187155 & 0.328719 & -0.063158 & \\
\hline 6 & -0.988421 & 0.934443 & -0.351477 & \\
\hline 8 & 1.615113 & 1.024567 & 0.554346 & \\
\hline 8 & 2.058476 & -0.972806 & -0.233752 & \\
\hline 1 & -0.166549 & -0.934855 & -1.201258 & \\
\hline 1 & -1.261879 & -0.986239 & 1.398267 & \\
\hline 1 & -2.437153 & -1.843471 & 0.294733 & \\
\hline 1 & -3.193726 & 0.662372 & -0.306014 & \\
\hline 1 & -0.733458 & 1.980642 & -0.484606 & \\
\hline \multicolumn{5}{|l|}{ in-TS1j } \\
\hline \multicolumn{5}{|c|}{ bordinates (Angstroms) } \\
\hline Number & $\mathrm{X}$ & $\mathrm{Y}$ & & $\mathrm{Z}$ \\
\hline \multicolumn{5}{|c|}{ 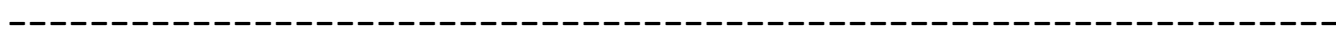 } \\
\hline 6 & -0.020268 & 0.915127 & 0.232378 & \\
\hline 6 & -1.276990 & -0.707265 & 0.980238 & \\
\hline 7 & 1.079903 & -0.040602 & -0.102872 & \\
\hline 6 & -1.814339 & -0.268052 & -0.261133 & \\
\hline 6 & -1.222556 & 0.947165 & -0.519826 & \\
\hline 8 & 0.910385 & -0.961322 & -0.895017 & \\
\hline 8 & 2.151307 & 0.223320 & 0.449350 & \\
\hline 1 & 0.463161 & 1.783009 & 0.675812 & \\
\hline 1 & -1.379810 & -0.077688 & 1.854907 & \\
\hline 1 & -1.089222 & -1.761829 & 1.195090 & \\
\hline 1 & -2.477518 & -0.853000 & -0.895077 & \\
\hline 1 & -1.564545 & 1.775899 & -1.135239 & \\
\hline \multicolumn{5}{|c|}{ out-TS1k } \\
\hline \multicolumn{5}{|c|}{-------- } \\
\hline Number & $\mathrm{X}$ & $\mathrm{Y}$ & & $\mathrm{z}$ \\
\hline & & 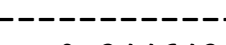 & & \\
\hline 6 & 0.176352 & -0.244643 & 0.571890 & \\
\hline 6 & -1.656269 & -0.871329 & -0.364598 & \\
\hline 6 & 1.588475 & -0.454742 & 0.204103 & \\
\hline 6 & -1.796531 & 0.528394 & -0.094469 & \\
\hline 8 & 2.217135 & 0.307462 & -0.512804 & \\
\hline 6 & -0.536283 & 0.957906 & 0.218622 & \\
\hline 1 & -0.169759 & -0.817999 & 1.427881 & \\
\hline 1 & -1.033369 & -1.186552 & -1.193034 & \\
\hline 1 & -2.410779 & -1.608700 & -0.072194 & \\
\hline
\end{tabular}




\begin{tabular}{|c|c|c|c|c|}
\hline 1 & 2.067770 & -1.369959 & 0.616474 & \\
\hline 1 & -2.737897 & 1.074472 & -0.068350 & \\
\hline 1 & -0.107506 & 1.955534 & 0.178371 & \\
\hline \multicolumn{5}{|l|}{ in-TS1k } \\
\hline Atomic & \multicolumn{4}{|c|}{ Coordinates (Angstroms) } \\
\hline Number & $\mathrm{x}$ & $\mathrm{Y}$ & & $\mathrm{Z}$ \\
\hline 6 & -0.182799 & -0.658528 & 0.471125 & \\
\hline 6 & 0.985255 & 1.147221 & 0.377227 & \\
\hline 6 & -1.189764 & 0.021763 & -0.375113 & \\
\hline 8 & -2.354471 & 0.173632 & -0.056341 & \\
\hline 6 & 1.703727 & 0.160862 & -0.375410 & \\
\hline 6 & 1.103740 & -1.026563 & -0.054636 & \\
\hline 1 & -0.632283 & -1.170810 & 1.327781 & \\
\hline 1 & 0.982258 & 1.084616 & 1.459610 & \\
\hline 1 & 0.781295 & 2.151202 & -0.004854 & \\
\hline 1 & -0.799778 & 0.355072 & -1.361105 & \\
\hline 1 & 2.496722 & 0.363638 & -1.092480 & \\
\hline 1 & 1.486591 & -2.041310 & -0.137375 & \\
\hline
\end{tabular}

out-TS11

\begin{tabular}{|c|c|c|c|}
\hline \multirow{2}{*}{$\begin{array}{l}\text { Atomic } \\
\text { Number }\end{array}$} & Coordinates & (Angstroms) & \multirow[b]{2}{*}{$\mathrm{Z}$} \\
\hline & $\mathrm{x}$ & $\mathrm{Y}$ & \\
\hline & 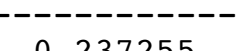 & 0197366 & --------1 \\
\hline 6 & 0.237255 & -0.197366 & 0.359886 \\
\hline 6 & -1.326076 & 1.133722 & -0.118781 \\
\hline 7 & 1.502990 & -0.430922 & -0.237384 \\
\hline 8 & 2.350412 & 0.426034 & 0.002391 \\
\hline 6 & -1.926018 & -0.168004 & -0.048263 \\
\hline 6 & -0.872357 & -1.038030 & -0.019218 \\
\hline 1 & 0.275124 & 0.300707 & 1.329137 \\
\hline 1 & -0.740570 & 1.401162 & -0.990886 \\
\hline 1 & -1.721776 & 1.994757 & 0.427733 \\
\hline 1 & -2.988199 & -0.369654 & 0.075171 \\
\hline 1 & -0.825627 & -2.100721 & -0.240340 \\
\hline \multicolumn{4}{|l|}{ in-TS11 } \\
\hline \multicolumn{4}{|l|}{-ーー-ーーーー- } \\
\hline Number & $\mathrm{X}$ & Y & Z \\
\hline & ---1 & -ーー-ー- & -ー-ー-ー- \\
\hline 6 & -0.218043 & -0.628415 & 0.447748 \\
\hline 6 & 0.901544 & 1.155328 & 0.330682 \\
\hline 7 & -1.085413 & 0.045939 & -0.475247 \\
\hline 8 & -2.240160 & 0.156163 & -0.074086 \\
\hline 6 & 1.690055 & 0.155674 & -0.341524 \\
\hline 6 & 1.088550 & -1.023281 & -0.020386 \\
\hline 1 & -0.753732 & -1.114055 & 1.272297 \\
\hline 1 & 0.878965 & 1.163994 & 1.415891 \\
\hline 1 & 0.662328 & 2.117613 & -0.125320 \\
\hline 1 & 2.509525 & 0.350170 & -1.029830 \\
\hline 1 & 1.449447 & -2.044424 & -0.112737 \\
\hline
\end{tabular}

$3 a$ 


$\begin{array}{lrrr}\text { Atomic } & \text { Coordinates } & \text { (Angstroms) } & \\ \text { Number } & \mathrm{X} & \mathrm{Y} & \\ - & 0.000000 & 0.722668 & 0.000000 \\ 6 & 0.000000 & -0.722668 & 0.000000 \\ 6 & 2.485829 & -1.128983 & 0.000000 \\ 6 & 3.509376 & -1.999593 & 0.000000 \\ 6 & 1.092174 & -1.531664 & 0.000000 \\ 6 & -3.509376 & 1.999593 & 0.000000 \\ 6 & -2.485829 & 1.128983 & 0.000000 \\ 6 & -1.092174 & 1.531664 & 0.000000 \\ 6 & 0.970328 & 1.213844 & 0.000000 \\ 1 & -0.970328 & -1.213844 & 0.000000 \\ 1 & 2.708853 & -0.063786 & 0.000000 \\ 1 & 3.341237 & -3.074452 & 0.000000 \\ 1 & 4.543089 & -1.667807 & 0.000000 \\ 1 & 0.920251 & -2.607658 & 0.000000 \\ 1 & -3.341237 & 3.074452 & 0.000000 \\ 1 & -4.543089 & 1.667807 & 0.000000 \\ 1 & -2.708853 & 0.063786 & 0.000000 \\ 1 & -0.920251 & 2.607658 & 0.000000 \\ 1 & & & \\ -1 & & & \end{array}$

\section{$3 b E$}

\begin{tabular}{|c|c|c|c|c|}
\hline \multirow{2}{*}{$\begin{array}{l}\text { Atomic } \\
\text { Number }\end{array}$} & Coordinates & \multicolumn{2}{|c|}{ (Angstroms) } & \multirow[b]{2}{*}{$\mathrm{Z}$} \\
\hline & $\mathrm{X}$ & $\mathrm{Y}$ & & \\
\hline 6 & -0.970868 & -0.539948 & -0.004705 & \\
\hline 6 & -0.298521 & 0.736324 & -0.003822 & \\
\hline 6 & 2.095864 & -0.057345 & 0.005017 & \\
\hline 6 & 3.409074 & 0.275979 & 0.005049 & \\
\hline 6 & 1.050011 & 0.933431 & -0.003792 & \\
\hline 6 & -4.678068 & -0.057429 & 0.005960 & \\
\hline 6 & -3.365417 & 0.233943 & 0.003903 & \\
\hline 6 & -2.313154 & -0.763208 & -0.001920 & \\
\hline 7 & 4.476241 & -0.601249 & -0.073850 & \\
\hline 1 & -0.334674 & -1.423225 & -0.009213 & \\
\hline 1 & -0.922286 & 1.626730 & -0.005176 & \\
\hline 1 & 1.825838 & -1.112404 & 0.006890 & \\
\hline 1 & 3.702580 & 1.324395 & 0.003243 & \\
\hline 1 & 1.394709 & 1.968213 & -0.008021 & \\
\hline 1 & -5.032481 & -1.086167 & 0.003131 & \\
\hline 1 & -5.436793 & 0.719247 & 0.010225 & \\
\hline 1 & -3.068078 & 1.281401 & 0.006829 & \\
\hline 1 & -2.651699 & -1.799008 & -0.004467 & \\
\hline 1 & 4.272204 & -1.571155 & 0.135909 & \\
\hline 1 & 5.343472 & -0.289765 & 0.343460 & \\
\hline \multicolumn{5}{|l|}{$3 \mathrm{bz}$} \\
\hline \multicolumn{5}{|c|}{ Coordinates (Angstroms) } \\
\hline Number & $\mathrm{X}$ & $\mathrm{Y}$ & & $\mathrm{Z}$ \\
\hline 6 & 1.014981 & 0.673719 & -0.003614 & \\
\hline 6 & 0.179930 & -0.502372 & -0.007603 & \\
\hline 6 & -2.083041 & 0.605078 & 0.004909 & \\
\hline 6 & -3.439544 & 0.534748 & 0.005343 & \\
\hline
\end{tabular}




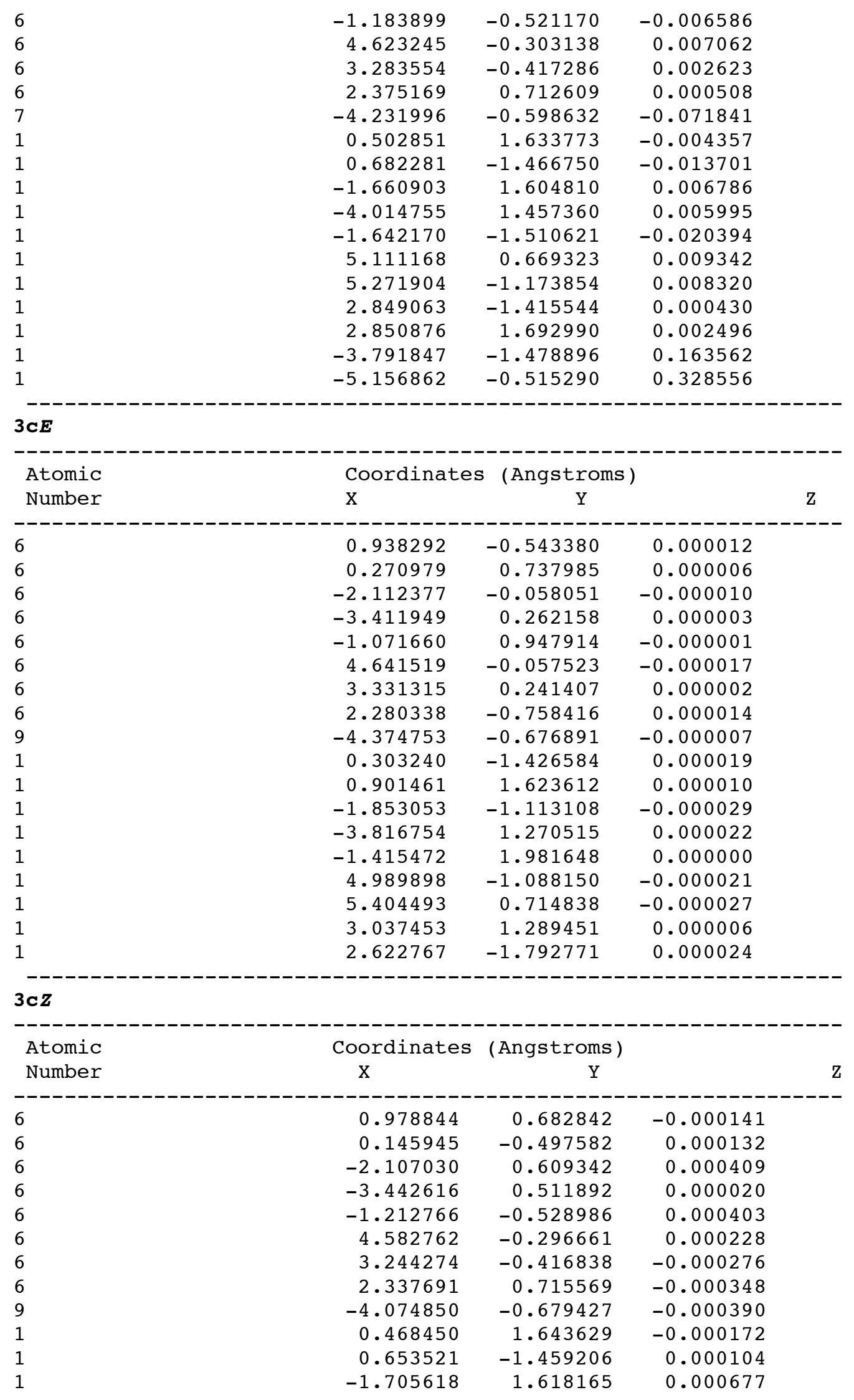




\begin{tabular}{|c|c|c|c|c|}
\hline 1 & -4.138021 & 1.344726 & -0.000008 & \\
\hline 1 & -1.697420 & -1.501795 & 0.000652 & \\
\hline 1 & 5.066470 & 0.677793 & 0.000582 & \\
\hline 1 & 5.234994 & -1.164585 & 0.000299 & \\
\hline 1 & 2.811815 & -1.415532 & -0.000587 & \\
\hline 1 & 2.816831 & 1.694181 & -0.000604 & \\
\hline \multicolumn{5}{|l|}{$3 \mathrm{~d} E$} \\
\hline Atomic & \multicolumn{4}{|c|}{ Coordinates (Angstroms) } \\
\hline Number & $\mathrm{x}$ & $\mathrm{Y}$ & & $\mathrm{Z}$ \\
\hline-- & ーーーーーーーーーー & -ー-ーーーー-ー & -ー-ー-ー-ー- & \\
\hline 6 & 1.456067 & -0.500145 & 0.000018 & \\
\hline 6 & 0.864277 & 0.817343 & -0.000035 & \\
\hline 6 & -1.559322 & 0.163365 & 0.000166 & \\
\hline 6 & -2.842871 & 0.556633 & -0.000084 & \\
\hline 6 & -0.463841 & 1.109833 & 0.000109 & \\
\hline 6 & 5.180279 & -0.222136 & -0.000185 & \\
\hline 6 & 3.889565 & 0.152019 & 0.000065 & \\
\hline 6 & 2.784687 & -0.787790 & 0.000126 & \\
\hline 17 & -4.182897 & -0.559762 & -0.000061 & \\
\hline 1 & 0.773705 & -1.347081 & 0.000004 & \\
\hline 1 & 1.546815 & 1.663728 & -0.000158 & \\
\hline 1 & -1.340862 & -0.900891 & 0.000417 & \\
\hline 1 & -3.157006 & 1.594642 & -0.000315 & \\
\hline 1 & -0.747418 & 2.161230 & 0.000147 & \\
\hline 1 & 5.468666 & -1.271088 & -0.000338 & \\
\hline 1 & 5.986402 & 0.505039 & -0.000245 & \\
\hline 1 & 3.656127 & 1.215031 & 0.000216 & \\
\hline 1 & 3.069766 & -1.839382 & 0.000224 & \\
\hline
\end{tabular}

\section{$3 d z$}

\begin{tabular}{|c|c|c|c|c|}
\hline Atomic & \multicolumn{4}{|c|}{ Coordinates (Angstroms) } \\
\hline Number & $\mathrm{x}$ & $\mathrm{Y}$ & & $\mathrm{Z}$ \\
\hline 6 & -1.533754 & 0.723501 & -0.000289 & \\
\hline 6 & -0.642371 & -0.412813 & 0.000092 & \\
\hline 6 & 1.549136 & 0.799102 & 0.000472 & \\
\hline 6 & 2.892260 & 0.839289 & 0.000190 & \\
\hline 6 & 0.717753 & -0.383160 & 0.000487 & \\
\hline 6 & -5.081305 & -0.442651 & -0.000094 & \\
\hline 6 & -3.738540 & -0.496276 & 0.000116 & \\
\hline 6 & -2.892623 & 0.682013 & -0.000230 & \\
\hline 17 & 3.927734 & -0.570395 & -0.000321 & \\
\hline 1 & -1.076384 & 1.710297 & -0.000650 & \\
\hline 1 & -1.103905 & -1.397389 & 0.000063 & \\
\hline 1 & 1.065946 & 1.773835 & 0.000767 & \\
\hline 1 & 3.456941 & 1.763233 & 0.000237 & \\
\hline 1 & 1.244340 & -1.333775 & 0.000842 & \\
\hline 1 & -5.612880 & 0.506518 & -0.000538 & \\
\hline 1 & -5.689509 & -1.341916 & 0.000169 & \\
\hline 1 & -3.256272 & -1.471883 & 0.000587 & \\
\hline 1 & -3.423093 & 1.633769 & -0.000483 & \\
\hline
\end{tabular}




\begin{tabular}{|c|c|c|c|c|}
\hline Number & $\mathrm{x}$ & $\mathrm{Y}$ & \multicolumn{2}{|r|}{$\mathrm{Z}$} \\
\hline 6 & 2.297228 & -0.460790 & 0.000080 & \\
\hline 6 & 1.770009 & 0.883648 & 0.000066 & \\
\hline 6 & -0.682697 & 0.347705 & 0.000177 & \\
\hline 6 & -1.945761 & 0.800950 & -0.000083 & \\
\hline 6 & 0.457573 & 1.240288 & 0.000122 & \\
\hline 6 & 6.030249 & -0.361539 & -0.000025 & \\
\hline 6 & 4.759041 & 0.074337 & -0.000147 & \\
\hline 6 & 3.610608 & -0.811617 & -0.000026 & \\
\hline 35 & -3.454380 & -0.351559 & -0.000031 & \\
\hline 1 & 1.574833 & -1.273793 & 0.000191 & \\
\hline 1 & 2.492649 & 1.696097 & -0.000002 & \\
\hline 1 & -0.513712 & -0.725396 & 0.000414 & \\
\hline 1 & -2.213379 & 1.851325 & -0.000343 & \\
\hline 1 & 0.225174 & 2.304122 & 0.000134 & \\
\hline 1 & 6.267500 & -1.423190 & 0.000184 & \\
\hline 1 & 6.870569 & 0.325841 & -0.000128 & \\
\hline 1 & 4.576856 & 1.147240 & -0.000325 & \\
\hline 1 & 3.845314 & -1.875568 & -0.000024 & \\
\hline \multicolumn{5}{|l|}{$3 e z$} \\
\hline \multirow{2}{*}{\multicolumn{5}{|c|}{ Atomic }} \\
\hline & & & & \\
\hline Number & $\mathrm{x}$ & $\mathrm{Y}$ & & $\mathrm{Z}$ \\
\hline \multicolumn{4}{|c|}{ 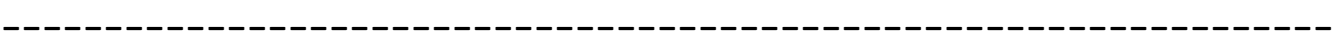 } & ---- \\
\hline 6 & -1.428031 & -0.321714 & 0.000078 & \\
\hline 6 & 0.699234 & 1.002018 & 0.000031 & \\
\hline 6 & 2.038030 & 1.109325 & -0.000023 & \\
\hline 6 & -0.071635 & -0.220441 & 0.000108 & \\
\hline 6 & -5.858352 & -0.591720 & -0.000157 & \\
\hline 6 & -4.514666 & -0.572587 & 0.000065 & \\
\hline 6 & -3.733878 & 0.649782 & 0.000049 & \\
\hline 35 & 3.234939 & -0.373870 & -0.000029 & \\
\hline 1 & -1.975931 & 1.774975 & -0.000043 & \\
\hline 1 & -1.835684 & -1.329765 & 0.000107 & \\
\hline 1 & 0.164563 & 1.950391 & -0.000001 & \\
\hline 1 & 2.560779 & 2.056791 & -0.000068 & \\
\hline 1 & 0.507859 & -1.139787 & 0.000203 & \\
\hline 1 & -6.440607 & 0.327224 & -0.000361 & \\
\hline 1 & -6.416967 & -1.522609 & -0.000143 & \\
\hline 1 & -3.980458 & -1.520654 & 0.000238 & \\
\hline 1 & -4.315195 & 1.571340 & 0.000064 & \\
\hline \multicolumn{5}{|l|}{$3 f E$} \\
\hline \multicolumn{5}{|c|}{ 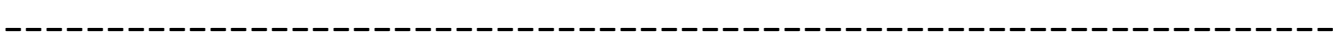 } \\
\hline $\begin{array}{l}\text { Atomic } \\
\text { Number }\end{array}$ & $\begin{array}{l}\text { Coordinat } \\
\mathrm{X}\end{array}$ & \multicolumn{3}{|c|}{ Coordinates (Angstroms) } \\
\hline & 071 & $0=050-7$ & 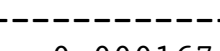 & \\
\hline 6 & 0.971454 & -0.535057 & -0.000167 & \\
\hline 6 & 0.319896 & 0.754671 & -0.000068 & \\
\hline 6 & -2.083856 & -0.002847 & 0.000001 & \\
\hline 6 & -3.392149 & 0.316478 & -0.000022 & \\
\hline 6 & -1.020727 & 0.981754 & -0.000168 & \\
\hline 6 & 4.681467 & -0.096321 & 0.000209 & \\
\hline 6 & 3.374663 & 0.217632 & 0.000146 & \\
\hline 6 & 2.310695 & -0.768340 & -0.000225 & \\
\hline
\end{tabular}




$\begin{array}{rrrr}6 & -4.522757 & -0.666536 & 0.000211 \\ 1 & 0.324907 & -1.409742 & -0.000267 \\ 1 & 0.963500 & 1.631237 & 0.000036 \\ 1 & -1.808752 & -1.056906 & 0.000190 \\ 1 & -3.670118 & 1.372057 & -0.000240 \\ 1 & -1.350443 & 2.020570 & -0.000350 \\ 1 & 5.018088 & -1.130905 & -0.000074 \\ 1 & 5.453304 & 0.667237 & 0.000540 \\ 1 & 3.093359 & 1.269164 & 0.000406 \\ 1 & 2.639743 & -1.807150 & -0.000543 \\ 1 & -5.167429 & -0.532382 & -0.879735 \\ 1 & -4.160594 & -1.700081 & 0.000663 \\ 1 & -5.167675 & -0.531695 & 0.879872 \\ \mathbf{3 f \boldsymbol { Z }} & & & \end{array}$

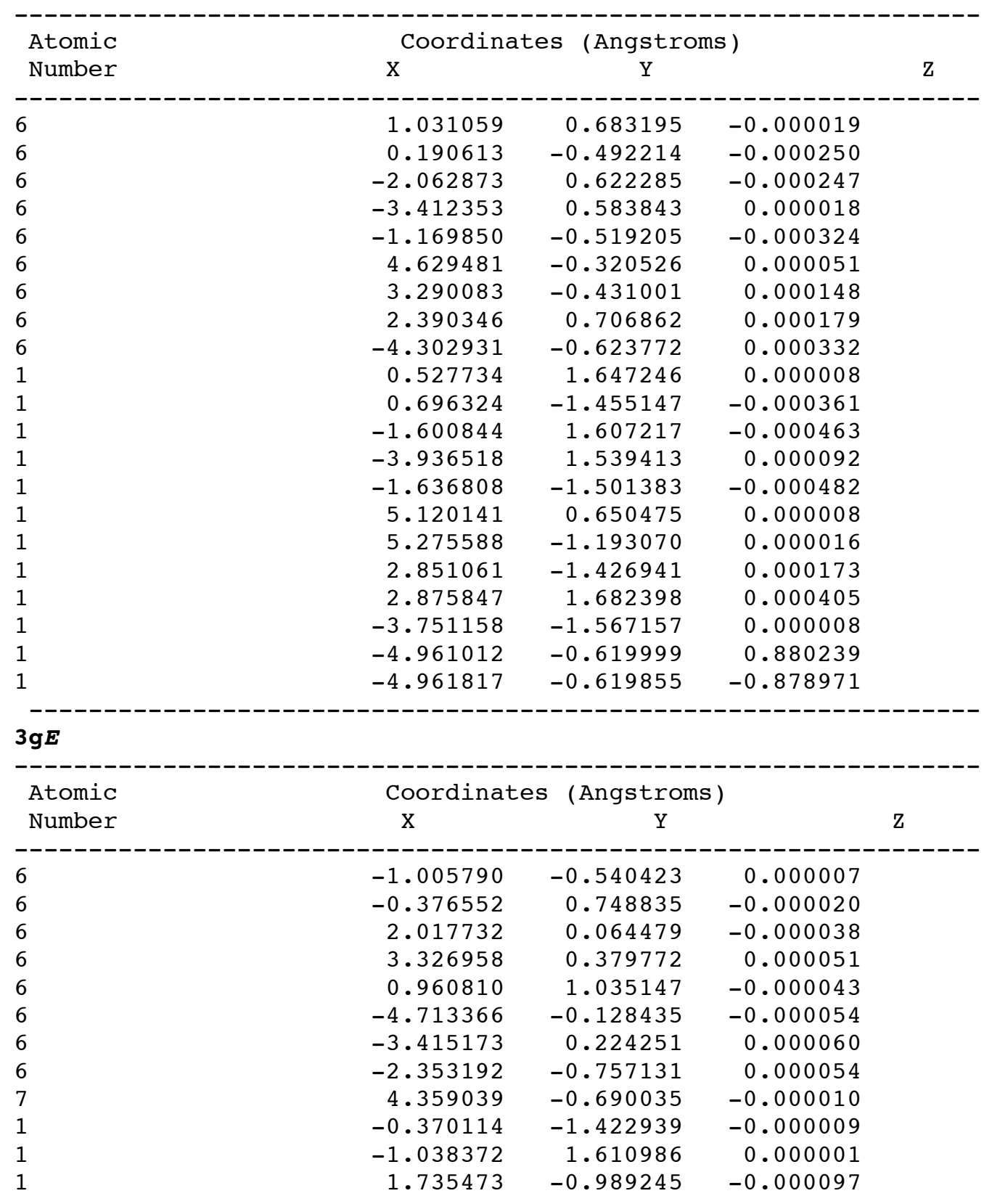




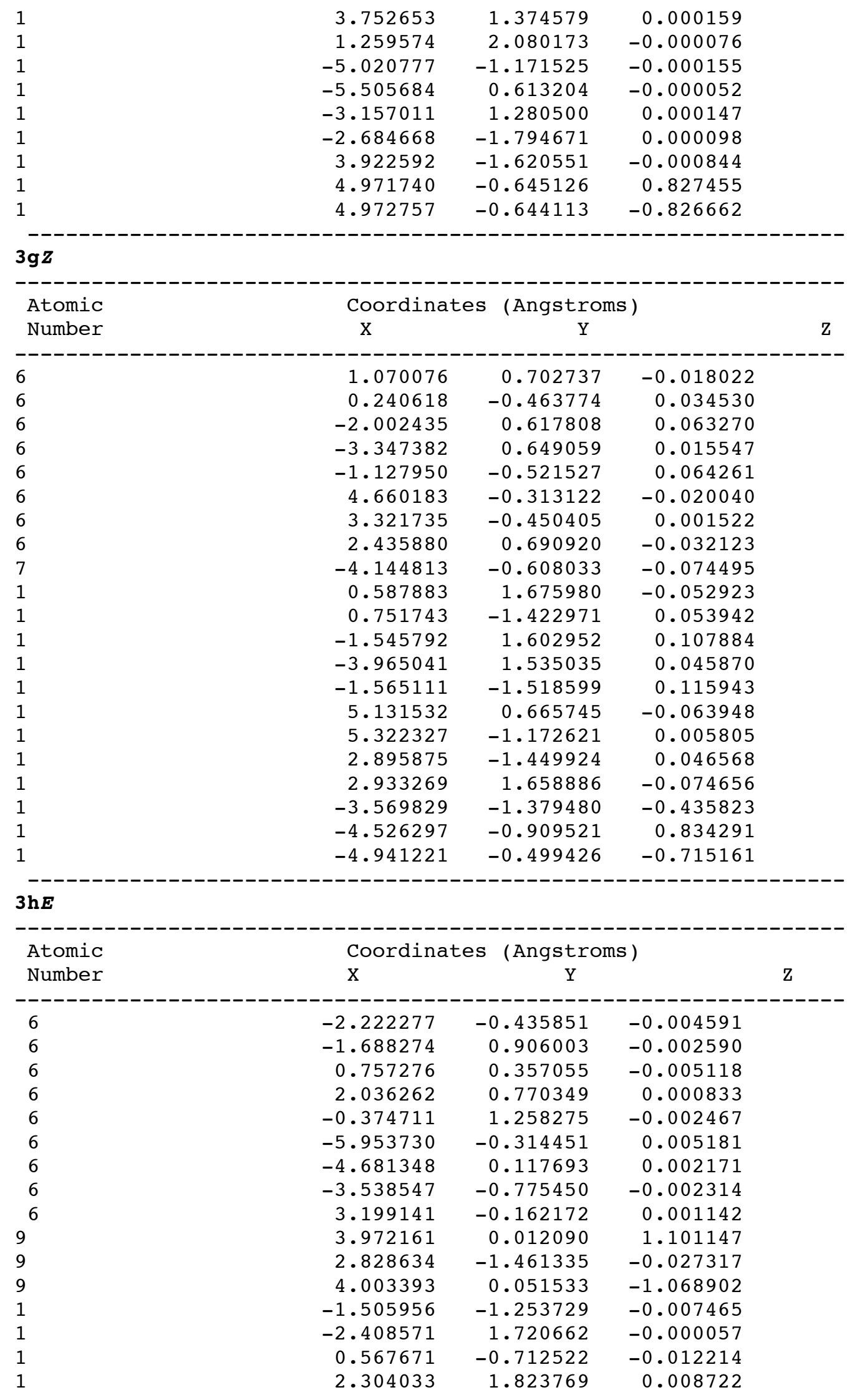




\begin{tabular}{rrrr}
1 & -0.135990 & 2.320539 & 0.000638 \\
1 & -6.193945 & -1.375387 & 0.004397 \\
1 & -6.791979 & 0.375364 & 0.008596 \\
1 & -4.494809 & 1.189684 & 0.003207 \\
1 & -3.780897 & -1.837667 & -0.003654 \\
\hline
\end{tabular}

\section{$3 h z$}

\begin{tabular}{|c|c|c|c|c|}
\hline \multirow{2}{*}{$\begin{array}{l}\text { Atomic } \\
\text { Number }\end{array}$} & \multirow{2}{*}{\multicolumn{3}{|c|}{$\mathrm{X}_{\mathrm{Y}}^{\text {Coordinates }}$ (Angstroms) }} & \multirow[b]{2}{*}{$\mathrm{Z}$} \\
\hline & & & & \\
\hline 6 & 2.344205 & 0.763718 & 0.000238 & \\
\hline 6 & 1.437265 & -0.360035 & 0.007197 & \\
\hline 6 & -0.732731 & 0.874874 & 0.002724 & \\
\hline 6 & -2.076130 & 0.990444 & -0.009876 & \\
\hline 6 & 0.076423 & -0.326983 & 0.004375 & \\
\hline 6 & 5.870337 & -0.464392 & -0.003920 & \\
\hline 6 & 4.526985 & -0.497597 & -0.000022 & \\
\hline 6 & 3.702169 & 0.695500 & -0.002118 & \\
\hline 6 & -3.110822 & -0.087311 & -0.003674 & \\
\hline 9 & -2.640246 & -1.323512 & -0.285051 & \\
\hline 9 & -3.730296 & -0.165963 & 1.200218 & \\
\hline 9 & -4.075958 & 0.185155 & -0.914714 & \\
\hline 1 & 1.906567 & 1.758873 & -0.004375 & \\
\hline 1 & 1.890611 & -1.348475 & 0.014154 & \\
\hline 1 & -0.192912 & 1.819238 & 0.013790 & \\
\hline 1 & -2.525201 & 1.979239 & 0.008160 & \\
\hline 1 & -0.447620 & -1.274691 & 0.008935 & \\
\hline 1 & 6.416370 & 0.476465 & -0.008564 & \\
\hline 1 & 6.464598 & -1.372892 & -0.002791 & \\
\hline 1 & 4.029557 & -1.465302 & 0.004215 & \\
\hline 1 & 4.250335 & 1.637114 & -0.007148 & \\
\hline \multicolumn{5}{|l|}{$3 i E$} \\
\hline 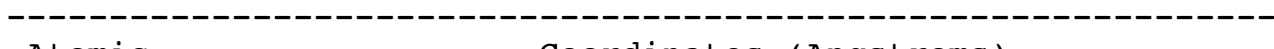 & \multicolumn{4}{|c|}{ Coordinates (Angstroms) } \\
\hline Number & $\mathrm{X}$ & $\mathrm{Y}$ & & Z \\
\hline & & --- & ---------- & \\
\hline 6 & -1.251928 & -0.507986 & -0.000054 & \\
\hline 6 & -0.678704 & 0.815023 & 0.000015 & \\
\hline 6 & 1.748815 & 0.204366 & 0.000299 & \\
\hline 6 & 3.047746 & 0.594719 & -0.000150 & \\
\hline 6 & 0.646910 & 1.131630 & 0.000192 & \\
\hline 6 & -4.977853 & -0.269347 & 0.000201 & \\
\hline 6 & -3.692872 & 0.124396 & -0.000246 & \\
\hline 6 & -2.579702 & -0.804393 & -0.000200 & \\
\hline 6 & 4.130189 & -0.327558 & -0.000072 & \\
\hline 7 & 5.027811 & -1.071114 & -0.000013 & \\
\hline 1 & -0.563006 & -1.349023 & 0.000028 & \\
\hline 1 & -1.374251 & 1.650766 & -0.000102 & \\
\hline 1 & 1.535069 & -0.861277 & 0.000701 & \\
\hline 1 & 3.311858 & 1.650145 & -0.000594 & \\
\hline 1 & 0.914526 & 2.187008 & 0.000278 & \\
\hline 1 & -5.250208 & -1.322437 & 0.000604 & \\
\hline 1 & -5.794700 & 0.445628 & 0.000179 & \\
\hline 1 & -3.473855 & 1.190161 & -0.000608 & \\
\hline 1 & -2.855719 & -1.858269 & -0.000297 & \\
\hline
\end{tabular}


$3 i z$

\begin{tabular}{|c|c|c|c|c|}
\hline Atomic & \multicolumn{3}{|c|}{ Coordinates (Angstroms) } & \\
\hline Number & $\mathrm{X}$ & $\mathrm{Y}$ & & $\mathrm{Z}$ \\
\hline \multicolumn{5}{|c|}{-1.348576} \\
\hline \multicolumn{5}{|c|}{-0.380681} \\
\hline \multicolumn{5}{|c|}{0.000300} \\
\hline \multicolumn{5}{|c|}{-0.000028} \\
\hline \multicolumn{5}{|c|}{-0.328874} \\
\hline \multicolumn{5}{|c|}{-4.868018} \\
\hline \multicolumn{5}{|c|}{-0.000214} \\
\hline \multicolumn{5}{|c|}{-0.000312} \\
\hline \multicolumn{5}{|c|}{-0.000201} \\
\hline \multicolumn{5}{|c|}{-0.000348} \\
\hline \multicolumn{5}{|c|}{-0.915873} \\
\hline \multicolumn{5}{|c|}{-1.373815} \\
\hline \multicolumn{5}{|c|}{1.227517} \\
\hline \multicolumn{5}{|c|}{-0.000175} \\
\hline \multicolumn{5}{|c|}{-1.271097} \\
\hline \multicolumn{5}{|c|}{-5.417151} \\
\hline \multicolumn{5}{|c|}{-1.420120} \\
\hline \multicolumn{5}{|c|}{-0.000302} \\
\hline 1 & -3.260055 & 1.597579 & -0.000626 & \\
\hline $3 \mathrm{j} E$ & & & & \\
\hline Atomic & Coordina & es (Angstrol & & \\
\hline Number & $\mathrm{x}$ & $\mathrm{Y}$ & & $\mathrm{Z}$ \\
\hline & -ーー-ー-ーー-ー & -ーーー-ーーーーー & -ーーーー-ーー & \\
\hline 6 & -1.755411 & 0.451511 & 0.000001 & \\
\hline 6 & -1.203309 & -0.879150 & 0.000066 & \\
\hline 6 & 1.221069 & -0.292785 & 0.000083 & \\
\hline 6 & 2.510113 & -0.682601 & 0.000014 & \\
\hline 6 & 0.118340 & -1.216099 & 0.000109 & \\
\hline 6 & -5.483893 & 0.267667 & -0.000053 & \\
\hline 6 & -4.205727 & -0.147987 & -0.000049 & \\
\hline 6 & -3.079803 & 0.764684 & -0.000052 & \\
\hline 7 & 3.581915 & 0.286220 & -0.000023 & \\
\hline 8 & 3.306426 & 1.490590 & 0.000163 & \\
\hline 8 & 4.728570 & -0.174396 & -0.000243 & \\
\hline 1 & -1.054387 & 1.282126 & -0.000002 & \\
\hline 1 & -1.910954 & -1.704727 & 0.000081 & \\
\hline 1 & 1.035774 & 0.777063 & 0.000110 & \\
\hline 1 & 2.872526 & -1.701814 & -0.000025 & \\
\hline 1 & 0.371349 & -2.274388 & 0.000173 & \\
\hline 1 & -5.737943 & 1.325256 & -0.000061 & \\
\hline 1 & -6.312796 & -0.433242 & -0.000049 & \\
\hline 1 & -4.003996 & -1.217034 & -0.000036 & \\
\hline 1 & -3.341218 & 1.822230 & -0.000105 & \\
\hline $3 \mathrm{j} z$ & & & & \\
\hline Atomic & Coordina & es (Angstror & & \\
\hline Number & $\mathrm{x}$ & $\mathrm{Y}$ & & $\mathrm{Z}$ \\
\hline & ---------0 & --ー-ー-ー-ー & --ー-ー-ー-ー & \\
\hline 6 & 1.874667 & 0.744269 & 0.000143 & \\
\hline 6 & 0.997861 & -0.398661 & 0.000287 & \\
\hline
\end{tabular}




\begin{tabular}{|c|c|c|c|c|}
\hline 6 & -1.189549 & 0.775705 & 0.000194 & \\
\hline 6 & -2.536896 & 0.899385 & 0.000059 & \\
\hline 6 & -0.368051 & -0.406080 & 0.000280 & \\
\hline 6 & 5.428549 & -0.398879 & -0.000247 & \\
\hline 6 & 4.086314 & -0.469757 & -0.000117 & \\
\hline 6 & 3.235440 & 0.703458 & -0.000072 & \\
\hline 7 & -3.496314 & -0.177963 & -0.000095 & \\
\hline 8 & -3.111547 & -1.353488 & 0.000030 & \\
\hline 8 & -4.684419 & 0.164258 & -0.000386 & \\
\hline 1 & 1.417201 & 1.730436 & 0.000204 & \\
\hline 1 & 1.475783 & -1.375482 & 0.000396 & \\
\hline 1 & -0.675829 & 1.734632 & 0.000246 & \\
\hline 1 & -3.036247 & 1.858142 & 0.000009 & \\
\hline 1 & -0.881778 & -1.358106 & 0.000338 & \\
\hline 1 & 5.947973 & 0.556826 & -0.000333 & \\
\hline 1 & 6.047697 & -1.290492 & -0.000274 & \\
\hline 1 & 3.614504 & -1.449926 & -0.000015 & \\
\hline 1 & 3.762618 & 1.656903 & -0.000226 & \\
\hline \multicolumn{5}{|l|}{$3 \mathbf{k} E$} \\
\hline Atomic & \multicolumn{4}{|c|}{ Coordinates (Angstroms) } \\
\hline Number & $\mathrm{X}$ & $\mathrm{Y}$ & & $\mathrm{z}$ \\
\hline 6 & -1.424021 & -0.522060 & 0.000263 & \\
\hline 6 & -0.811947 & 0.783362 & 0.000254 & \\
\hline 6 & 1.600171 & 0.104667 & 0.000560 & \\
\hline 6 & 2.915000 & 0.433018 & -0.000171 & \\
\hline 6 & 0.522974 & 1.060605 & 0.000420 & \\
\hline 6 & -5.141801 & -0.176160 & -0.000227 & \\
\hline 6 & -3.845834 & 0.179839 & -0.000421 & \\
\hline 6 & -2.759810 & -0.780508 & -0.000065 & \\
\hline 6 & 3.956223 & -0.593631 & -0.000001 & \\
\hline 8 & 5.154275 & -0.365838 & -0.000584 & \\
\hline 1 & -0.759583 & -1.382532 & 0.000577 & \\
\hline 1 & -1.483038 & 1.638945 & 0.000082 & \\
\hline 1 & 1.346395 & -0.955258 & 0.001230 & \\
\hline 1 & 3.245886 & 1.469718 & -0.000902 & \\
\hline 1 & 0.819630 & 2.107960 & 0.000465 & \\
\hline 1 & -5.444980 & -1.220835 & 0.000194 & \\
\hline 1 & -5.937343 & 0.562444 & -0.000483 & \\
\hline 1 & -3.595883 & 1.238783 & -0.000809 & \\
\hline 1 & -3.066006 & -1.826059 & -0.000072 & \\
\hline 1 & 3.574992 & -1.641257 & 0.000727 & \\
\hline \multicolumn{5}{|l|}{$3 \mathbf{k} z$} \\
\hline Atomic & \multicolumn{4}{|c|}{ Coordinates (Angstroms) } \\
\hline Number & $\mathrm{X}$ & $\mathrm{Y}$ & & $\mathrm{Z}$ \\
\hline & & & ・ーーーーーーー & \\
\hline 6 & -1.511787 & 0.698060 & 0.000192 & \\
\hline 6 & -0.653741 & -0.461196 & 0.000315 & \\
\hline 6 & 1.569905 & 0.680768 & 0.000088 & \\
\hline 6 & 2.929664 & 0.697835 & -0.000095 & \\
\hline 6 & 0.709943 & -0.478504 & 0.000263 & \\
\hline 6 & -5.086661 & -0.380027 & -0.000361 & \\
\hline 6 & -3.745897 & -0.471948 & -0.000018 & \\
\hline 6 & -2.872188 & 0.685141 & 0.000038 & \\
\hline
\end{tabular}




\begin{tabular}{|c|c|c|c|}
\hline 6 & 3.810249 & -0.472333 & -0.000125 \\
\hline 8 & 5.028606 & -0.389882 & -0.000229 \\
\hline 1 & -1.032983 & 1.673925 & 0.000221 \\
\hline 1 & -1.146380 & -1.430650 & 0.000467 \\
\hline 1 & 1.075240 & 1.650016 & 0.000103 \\
\hline 1 & 3.452530 & 1.651330 & -0.000230 \\
\hline 1 & 1.186923 & -1.454318 & 0.000358 \\
\hline 1 & -5.590831 & 0.583841 & -0.000607 \\
\hline 1 & -5.720073 & -1.261638 & -0.000404 \\
\hline 1 & -3.290791 & -1.460232 & 0.000223 \\
\hline 1 & -3.380539 & 1.648754 & -0.000046 \\
\hline 1 & 3.321133 & -1.468746 & -0.000028 \\
\hline \multicolumn{4}{|l|}{$31 E$} \\
\hline Atomic & \multicolumn{3}{|c|}{ Coordinates (Angstroms) } \\
\hline Number & $\mathrm{x}$ & $\mathrm{Y}$ & \\
\hline 6 & 1.404625 & -0.529202 & 0.000157 \\
\hline 6 & 0.783551 & 0.769175 & 0.000124 \\
\hline 6 & -1.613144 & 0.067047 & 0.000222 \\
\hline 6 & -2.935894 & 0.376247 & -0.000034 \\
\hline 6 & -0.556315 & 1.036053 & 0.000160 \\
\hline 6 & 5.118947 & -0.149096 & -0.000174 \\
\hline 6 & 3.820230 & 0.197743 & -0.000152 \\
\hline 6 & 2.744334 & -0.772779 & 0.000025 \\
\hline 7 & -3.848990 & -0.692021 & 0.000070 \\
\hline 8 & -5.030178 & -0.335429 & -0.000318 \\
\hline 1 & 0.748208 & -1.395463 & 0.000303 \\
\hline 1 & 1.446855 & 1.630796 & 0.000057 \\
\hline 1 & -1.372926 & -0.993848 & 0.000467 \\
\hline 1 & -3.335203 & 1.391312 & -0.000308 \\
\hline 1 & -0.861969 & 2.080560 & 0.000145 \\
\hline 1 & 5.429460 & -1.191537 & -0.000061 \\
\hline 1 & 5.909099 & 0.595211 & -0.000305 \\
\hline 1 & 3.561988 & 1.254603 & -0.000260 \\
\hline 1 & 3.060837 & -1.815189 & 0.000053 \\
\hline \multirow{2}{*}{\multicolumn{4}{|c|}{$31 z$}} \\
\hline & & & \\
\hline \multirow{2}{*}{$\begin{array}{l}\text { Atomic } \\
\text { Number }\end{array}$} & \multicolumn{3}{|c|}{ Coordinates (Angstroms) } \\
\hline & $\mathrm{X}$ & $\mathrm{Y}$ & Z \\
\hline 6 & 1.479201 & 0.703809 & 0.000013 \\
\hline 6 & 0.617622 & -0.450268 & 0.000064 \\
\hline 6 & -1.595834 & 0.698025 & 0.000049 \\
\hline 6 & -2.958759 & 0.672353 & 0.000005 \\
\hline 6 & -0.747973 & -0.461810 & 0.000081 \\
\hline 6 & 5.049506 & -0.387963 & -0.000053 \\
\hline 6 & 3.708247 & -0.476335 & -0.000018 \\
\hline 6 & 2.840469 & 0.684330 & -0.000021 \\
\hline 7 & -3.634687 & -0.558141 & -0.000002 \\
\hline 8 & -4.863350 & -0.445467 & -0.000098 \\
\hline 1 & 1.004903 & 1.682070 & 0.000002 \\
\hline 1 & 1.104473 & -1.422633 & 0.000092 \\
\hline 1 & -1.126914 & 1.679339 & 0.000058 \\
\hline 1 & -3.570853 & 1.573868 & -0.000021 \\
\hline 1 & -1.256261 & -1.420748 & 0.000121 \\
\hline
\end{tabular}




$\begin{array}{rrrr}1 & 5.556263 & 0.574521 & -0.000088 \\ 1 & 5.680364 & -1.271348 & -0.000047 \\ 1 & 3.249636 & -1.462840 & 0.000012 \\ 1 & 3.353121 & 1.645653 & -0.000053\end{array}$

$(z, z, z, z) 3 \mathrm{~m}$

\begin{tabular}{|c|c|c|c|c|}
\hline Atomic & Coordinates & \multicolumn{3}{|c|}{ (Angstroms) } \\
\hline Number & $\mathrm{X}$ & $\mathrm{Y}$ & & $\mathrm{Z}$ \\
\hline 6 & -0.446355 & 0.567831 & -0.000063 & \\
\hline 6 & 0.446355 & -0.567824 & -0.000052 & \\
\hline 6 & 2.647983 & 0.646075 & -0.000004 & \\
\hline 6 & 3.997882 & 0.669762 & 0.000039 & \\
\hline 6 & 1.807031 & -0.534285 & -0.000048 & \\
\hline 6 & -3.997879 & -0.669765 & 0.000039 & \\
\hline 6 & -2.647980 & -0.646072 & -0.000024 & \\
\hline 6 & -1.807031 & 0.534291 & -0.000074 & \\
\hline 6 & 4.942228 & -0.496474 & 0.000056 & \\
\hline 6 & -4.942232 & 0.496465 & 0.000073 & \\
\hline 1 & 0.014735 & 1.552806 & -0.000096 & \\
\hline 1 & -0.014736 & -1.552798 & -0.000081 & \\
\hline 1 & 2.141617 & 1.609087 & 0.000019 & \\
\hline 1 & 4.478014 & 1.648224 & 0.000108 & \\
\hline 1 & 2.317354 & -1.494757 & -0.000067 & \\
\hline 1 & -4.478006 & -1.648229 & 0.000106 & \\
\hline 1 & -2.141611 & -1.609082 & -0.000011 & \\
\hline 1 & -2.317355 & 1.494762 & -0.000113 & \\
\hline 1 & 4.432822 & -1.463365 & 0.000032 & \\
\hline 1 & 5.599989 & -0.463751 & -0.879572 & \\
\hline 1 & 5.599889 & -0.463767 & 0.879750 & \\
\hline 1 & -5.599919 & 0.463717 & 0.879746 & \\
\hline 1 & -4.432834 & 1.463360 & 0.000102 & \\
\hline 1 & -5.599967 & 0.463772 & -0.879576 & \\
\hline
\end{tabular}

$(E, Z, Z, E) 3 \mathrm{~m}$

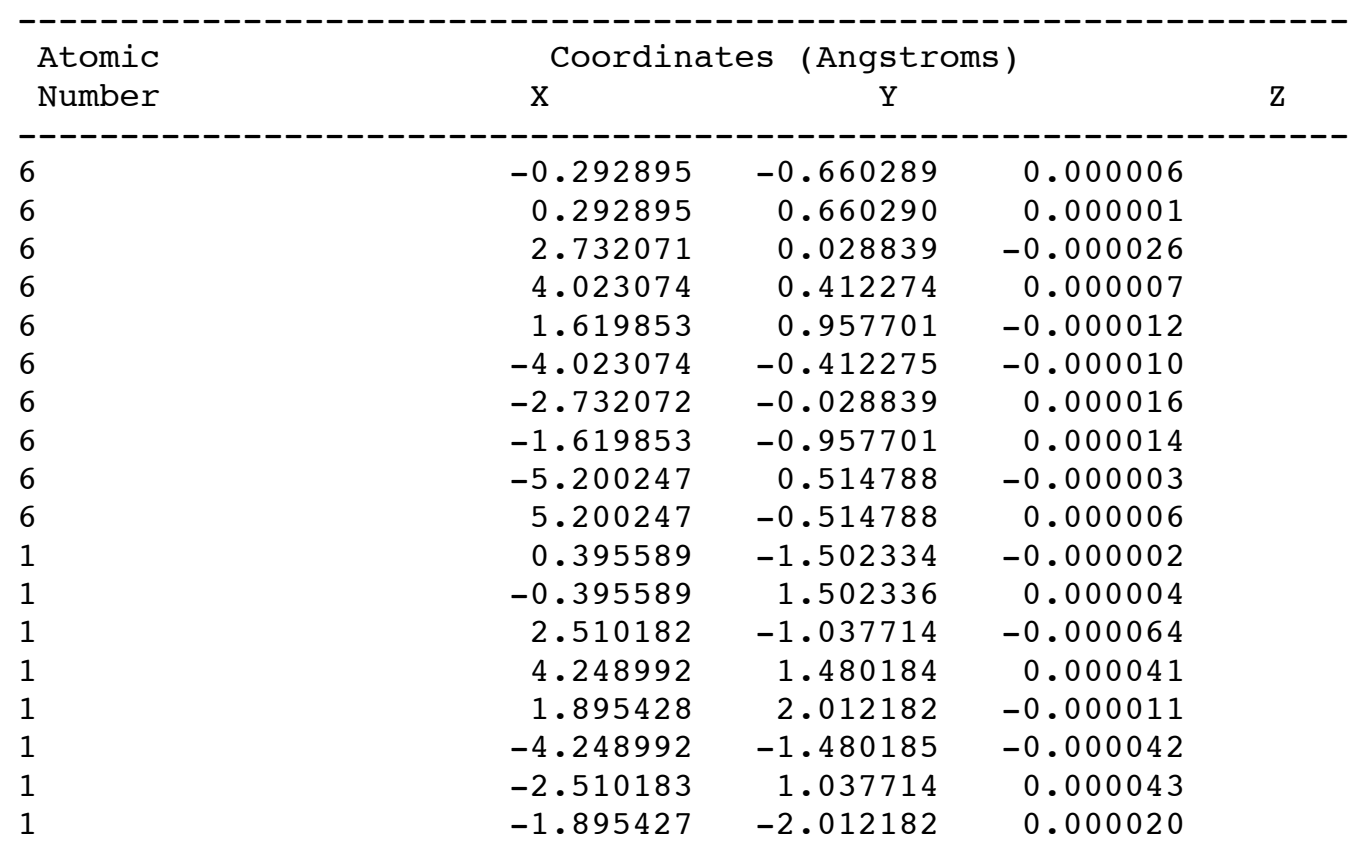




\begin{tabular}{rrrr}
1 & -4.887973 & 1.564560 & 0.000170 \\
1 & -5.837915 & 0.350169 & -0.879949 \\
1 & -5.838096 & 0.349933 & 0.879767 \\
1 & 4.887973 & -1.564560 & -0.000179 \\
1 & 5.838106 & -0.349926 & -0.879755 \\
1 & 5.837905 & -0.350175 & 0.879961 \\
\hline
\end{tabular}

$(E, Z, Z, Z) 3 m$

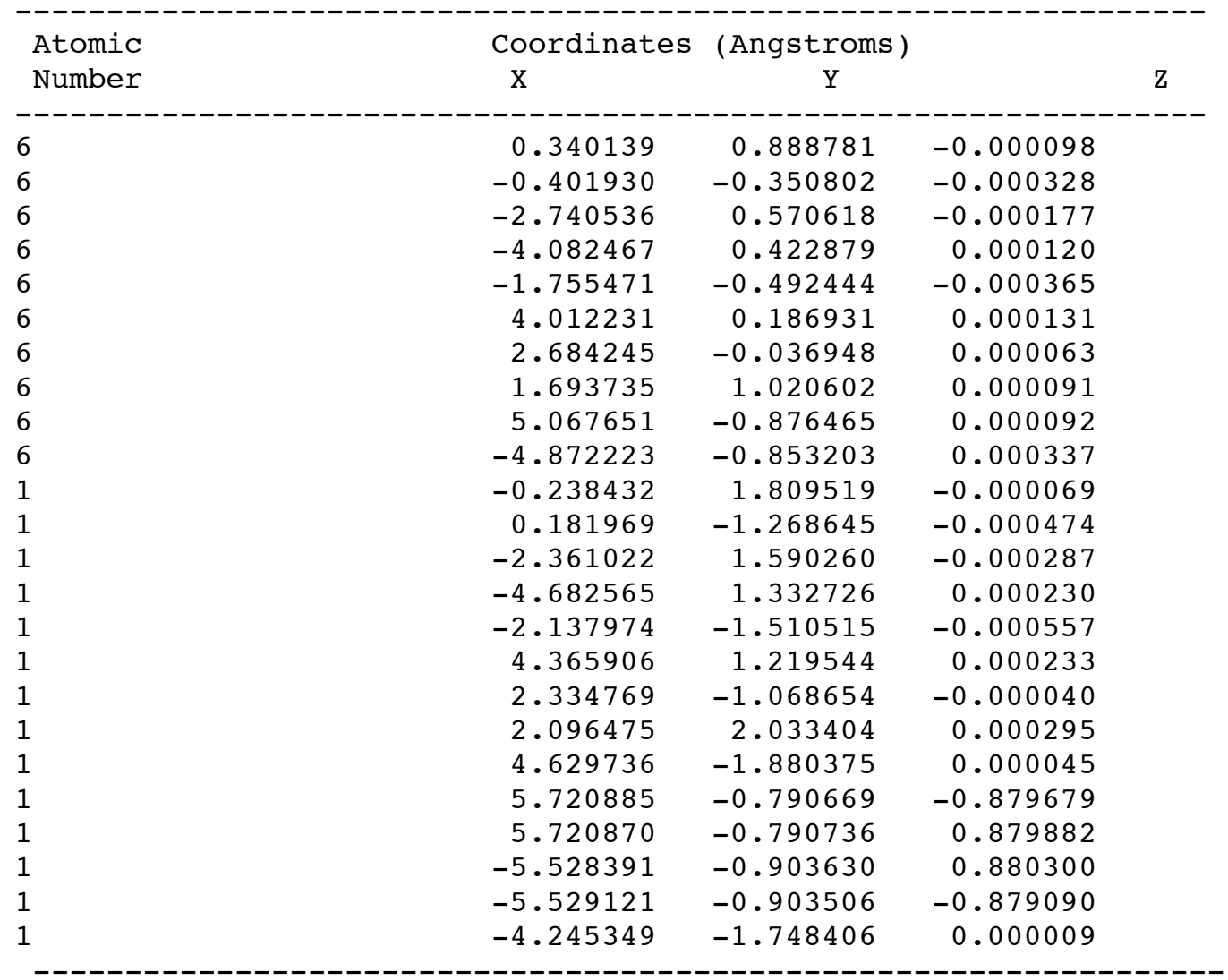

Tsa

\begin{tabular}{|c|c|c|c|c|}
\hline Atomic & \multicolumn{4}{|c|}{ Coordinates (Angstroms) } \\
\hline Number & $\mathrm{x}$ & $\mathrm{Y}$ & & $\mathrm{Z}$ \\
\hline 6 & -0.877429 & -1.426410 & 0.791357 & \\
\hline 6 & 0.877343 & -1.426522 & -0.791284 & \\
\hline 6 & -1.798587 & -0.699036 & 0.073219 & \\
\hline 6 & -1.722808 & 0.674460 & -0.251889 & \\
\hline 6 & -0.694594 & 1.606803 & -0.155763 & \\
\hline 6 & 0.694652 & 1.606763 & 0.155833 & \\
\hline 6 & 1.722884 & 0.674419 & 0.251745 & \\
\hline 6 & 1.798542 & -0.699119 & -0.073228 & \\
\hline 1 & -0.175035 & -0.930374 & 1.445061 & \\
\hline 1 & -1.054707 & -2.479481 & 0.992922 & \\
\hline 1 & 0.174998 & -0.930515 & -1.445060 & \\
\hline 1 & 1.054564 & -2.479624 & -0.992751 & \\
\hline 1 & -2.653467 & -1.233697 & -0.341844 & \\
\hline 1 & -2.647884 & 1.088683 & -0.653428 & \\
\hline
\end{tabular}




$\begin{array}{rrrr}1 & -1.047085 & 2.621032 & -0.350141 \\ 1 & 1.047114 & 2.620956 & 0.350462 \\ 1 & 2.648114 & 1.088663 & 0.652911 \\ 1 & 2.653373 & -1.233790 & 0.341926\end{array}$

\section{TS'a}

\begin{tabular}{|c|c|c|c|c|}
\hline Atomic & \multicolumn{4}{|c|}{ Coordinates (Angstroms) } \\
\hline Number & $\mathrm{X}$ & $\mathrm{Y}$ & & $\mathrm{Z}$ \\
\hline 6 & -0.122164 & 1.689127 & -0.450060 & \\
\hline 6 & -1.736205 & -0.554675 & 0.591512 & \\
\hline 6 & -0.519791 & -1.222887 & 0.601338 & \\
\hline 6 & 0.347867 & -1.402569 & -0.520038 & \\
\hline 6 & 1.623390 & -0.869164 & -0.448495 & \\
\hline 6 & 1.809775 & 0.286055 & 0.351259 & \\
\hline 6 & 0.888225 & 1.353808 & 0.453022 & \\
\hline 6 & -2.061695 & 0.316542 & -0.445064 & \\
\hline 1 & -0.040146 & 1.308463 & -1.460780 & \\
\hline 1 & -0.621077 & 2.646502 & -0.339597 & \\
\hline 1 & -2.265836 & -0.449532 & 1.537694 & \\
\hline 1 & -0.072963 & -1.404302 & 1.578267 & \\
\hline 1 & -0.001951 & -1.938234 & -1.405371 & \\
\hline 1 & 2.463978 & -1.287807 & -1.006461 & \\
\hline 1 & 2.780408 & 0.448671 & 0.826898 & \\
\hline 1 & 1.042717 & 2.035662 & 1.291377 & \\
\hline 1 & -1.721257 & 0.110293 & -1.452935 & \\
\hline 1 & -2.940285 & 0.952872 & -0.369936 & \\
\hline
\end{tabular}

out-TSb

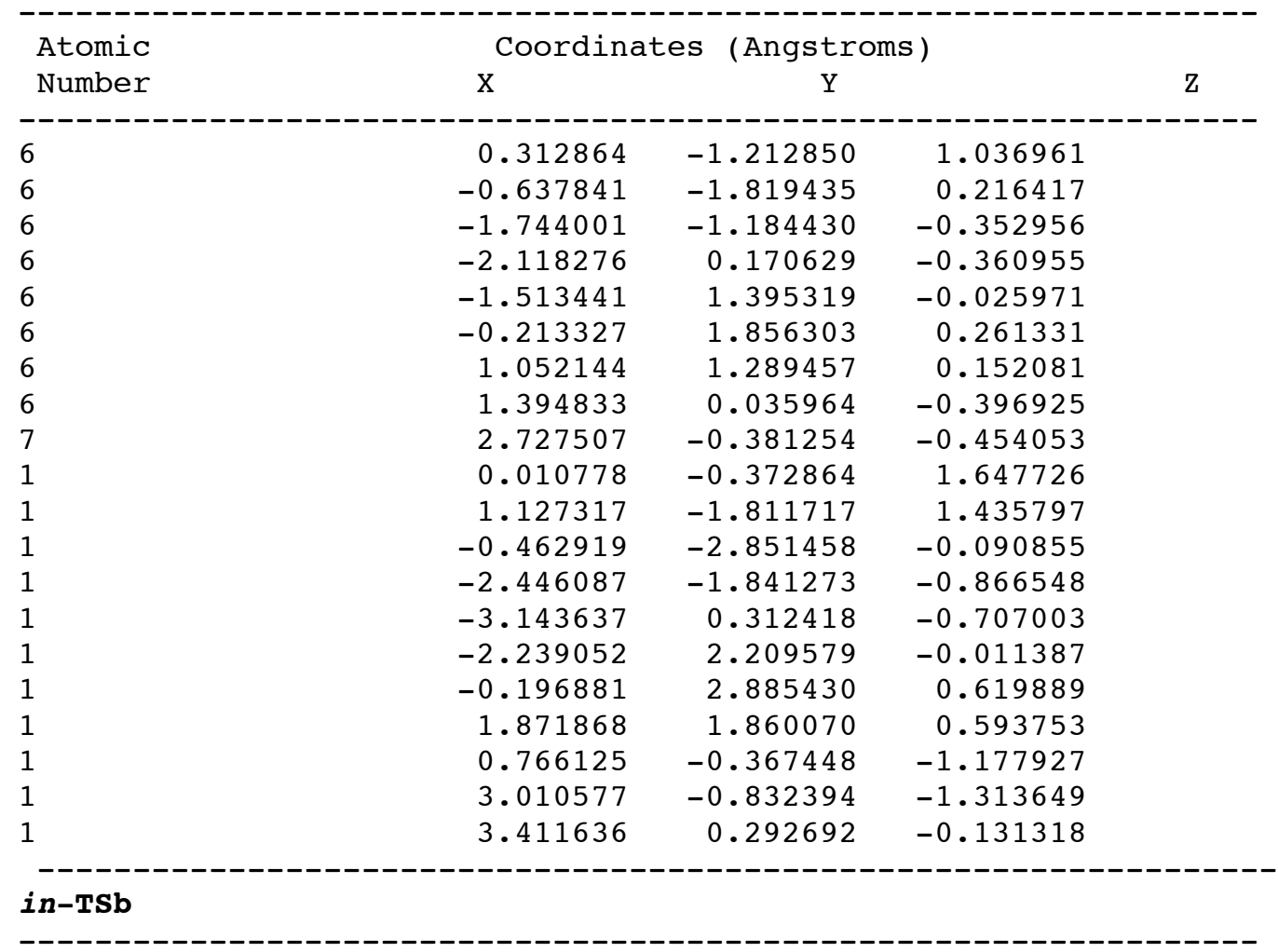




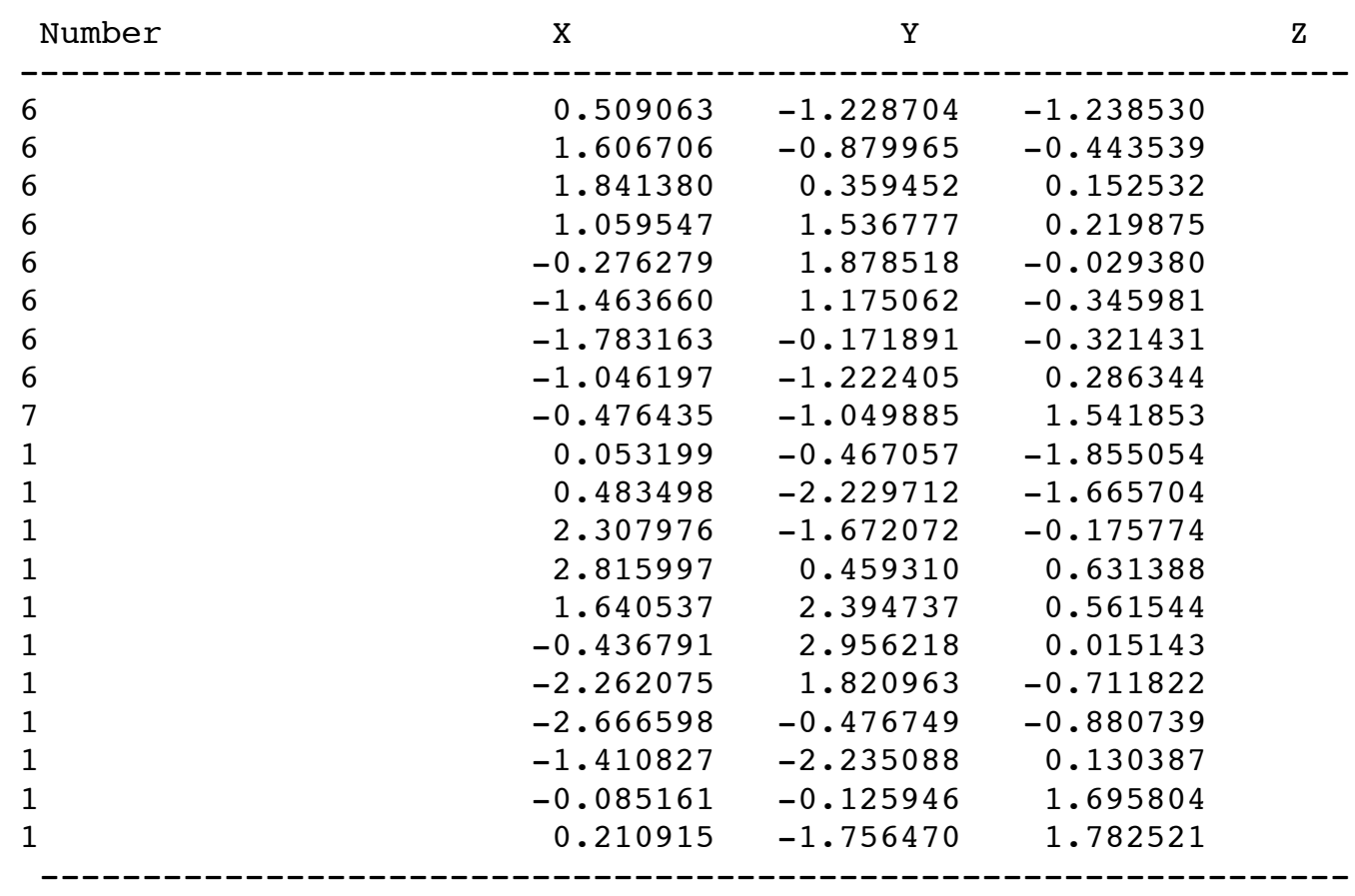

\section{out-TSC}

\begin{tabular}{|c|c|c|c|c|}
\hline Atomic & \multicolumn{4}{|c|}{ Coordinates (Angstroms) } \\
\hline Number & $\mathrm{X}$ & $\mathrm{Y}$ & & $\mathrm{Z}$ \\
\hline 6 & 0.244169 & -1.341562 & 1.035130 & \\
\hline 6 & -0.718584 & -1.822229 & 0.172953 & \\
\hline 6 & -1.789762 & -1.084216 & -0.370606 & \\
\hline 6 & -2.074905 & 0.280041 & -0.352548 & \\
\hline 6 & -1.404645 & 1.470840 & 0.030478 & \\
\hline 6 & -0.093746 & 1.865270 & 0.297251 & \\
\hline 6 & 1.153694 & 1.231237 & 0.153935 & \\
\hline 6 & 1.403249 & 0.018155 & -0.449803 & \\
\hline 9 & 2.683699 & -0.410920 & -0.501070 & \\
\hline 1 & 0.046612 & -0.464292 & 1.635187 & \\
\hline 1 & 1.029280 & -2.000612 & 1.396269 & \\
\hline 1 & -0.615568 & -2.846575 & -0.185844 & \\
\hline 1 & -2.537663 & -1.683634 & -0.889346 & \\
\hline 1 & -3.083408 & 0.494553 & -0.709151 & \\
\hline 1 & -2.100051 & 2.308030 & 0.100480 & \\
\hline 1 & -0.017550 & 2.882818 & 0.678115 & \\
\hline 1 & 2.014448 & 1.713265 & 0.614277 & \\
\hline 1 & 0.793796 & -0.410486 & -1.231099 & \\
\hline \multicolumn{5}{|l|}{ in-TSc } \\
\hline Atomic & \multicolumn{4}{|c|}{ Coordinates (Angstroms) } \\
\hline Number & $\mathrm{X}$ & $\mathrm{Y}$ & & $\mathrm{Z}$ \\
\hline 6 & 0.763907 & -1.214730 & -1.196118 & \\
\hline 6 & 1.720742 & -0.693507 & -0.349832 & \\
\hline 6 & 1.767101 & 0.616785 & 0.169831 & \\
\hline 6 & 0.837365 & 1.654987 & 0.187435 & \\
\hline 6 & -0.541063 & 1.803521 & -0.102365 & \\
\hline 6 & -1.619577 & 0.955118 & -0.357627 & \\
\hline
\end{tabular}




\begin{tabular}{|c|c|c|c|c|}
\hline 6 & -1.762738 & -0.439327 & -0.275317 & \\
\hline 6 & -0.923059 & -1.331288 & 0.358463 & \\
\hline 9 & -0.218198 & -0.939869 & 1.437380 & \\
\hline 1 & 0.167060 & -0.556716 & -1.811917 & \\
\hline 1 & 0.868800 & -2.233969 & -1.560234 & \\
\hline 1 & 2.498618 & -1.366821 & 0.010357 & \\
\hline 1 & 2.714947 & 0.874677 & 0.643109 & \\
\hline 1 & 1.279110 & 2.599801 & 0.507186 & \\
\hline 1 & -0.829945 & 2.854818 & -0.137264 & \\
\hline 1 & -2.509137 & 1.471585 & -0.717335 & \\
\hline 1 & -2.575752 & -0.892486 & -0.841247 & \\
\hline 1 & -1.105987 & -2.401428 & 0.364097 & \\
\hline \multicolumn{5}{|l|}{ out-TSd } \\
\hline Atomic & \multicolumn{3}{|c|}{ Coordinates (Angstroms) } & \\
\hline Number & $\mathrm{X}$ & $\mathrm{Y}$ & & $\mathrm{Z}$ \\
\hline \multicolumn{5}{|c|}{ 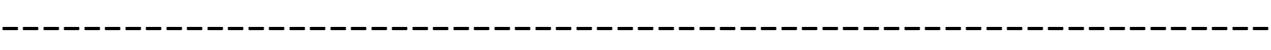 } \\
\hline 6 & -1.118230 & -1.256008 & -0.150634 & \\
\hline 6 & -1.120997 & -1.250952 & 1.228789 & \\
\hline 6 & 0.017537 & -1.248607 & 2.058696 & \\
\hline 6 & 1.372522 & -1.084053 & 1.772827 & \\
\hline 6 & 2.154078 & -0.706104 & 0.651596 & \\
\hline 6 & 1.950701 & -0.076107 & -0.577223 & \\
\hline 6 & 0.852298 & 0.586576 & -1.155231 & \\
\hline 6 & -0.340073 & 0.893170 & -0.527730 & \\
\hline 17 & -1.564170 & 1.811924 & -1.401218 & \\
\hline 1 & -0.412772 & 1.036206 & 0.538193 & \\
\hline 1 & -0.252177 & -1.612842 & -0.690144 & \\
\hline 1 & -2.057275 & -1.262296 & -0.696434 & \\
\hline 1 & 0.933862 & 0.828853 & -2.212786 & \\
\hline 1 & 2.833720 & -0.079712 & -1.215140 & \\
\hline 1 & 3.206159 & -0.945877 & 0.810076 & \\
\hline 1 & 2.004977 & -1.325450 & 2.628158 & \\
\hline 1 & -0.190595 & -1.416029 & 3.115284 & \\
\hline 1 & -2.082019 & -1.173048 & 1.736951 & \\
\hline \multicolumn{5}{|l|}{ in-TSd } \\
\hline Atomic & \multicolumn{3}{|c|}{ Coordinates (Angstroms) } & \\
\hline Number & $\mathrm{X}$ & $\mathrm{Y}$ & & $\mathrm{Z}$ \\
\hline \multicolumn{4}{|l|}{--------} & \\
\hline 6 & -1.394437 & -1.180067 & -0.721340 & \\
\hline 6 & -1.363409 & -1.238478 & 0.656885 & \\
\hline 6 & -0.219339 & -1.306030 & 1.475151 & \\
\hline 6 & 1.140615 & -1.127627 & 1.215195 & \\
\hline 6 & 1.927997 & -0.646195 & 0.144401 & \\
\hline 6 & 1.709310 & 0.001036 & -1.075789 & \\
\hline 6 & 0.577038 & 0.589153 & -1.654138 & \\
\hline 6 & -0.617652 & 0.985261 & -1.071693 & \\
\hline 1 & -1.414465 & 1.372519 & -1.696073 & \\
\hline 17 & -0.671352 & 1.718541 & 0.518953 & \\
\hline 1 & -0.557299 & -1.542723 & -1.299748 & \\
\hline 1 & -2.352796 & -1.169336 & -1.234409 & \\
\hline 1 & 0.587563 & 0.667421 & -2.743010 & \\
\hline 1 & 2.584088 & 0.003987 & -1.726087 & \\
\hline 1 & 2.985818 & -0.858142 & 0.305898 & \\
\hline 1 & 1.763437 & -1.436200 & 2.055636 & \\
\hline
\end{tabular}




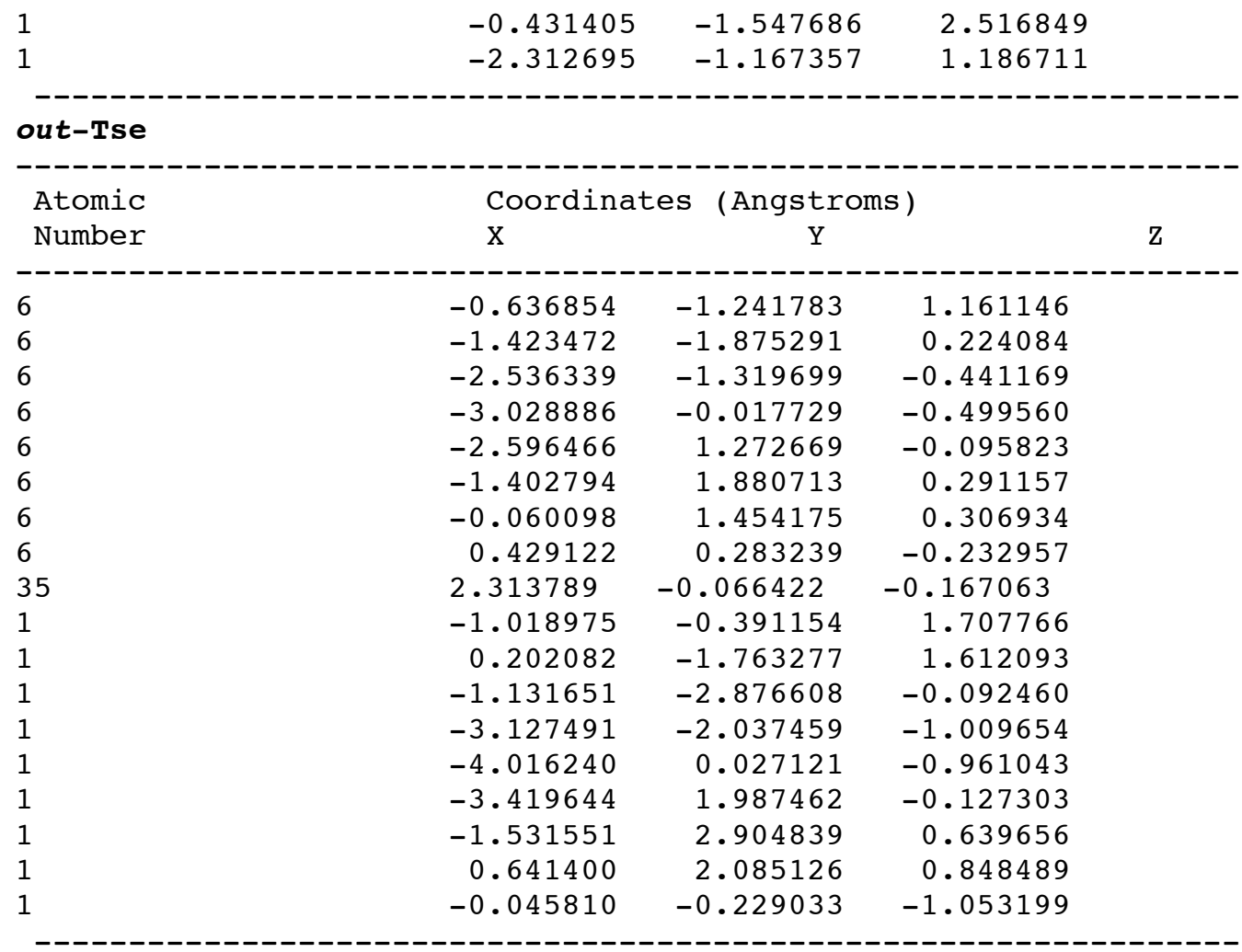

\section{in-Tse}

\begin{tabular}{|c|c|c|c|c|}
\hline \multirow{2}{*}{$\begin{array}{l}\text { Atomic } \\
\text { Number }\end{array}$} & \multicolumn{3}{|c|}{ Coordinates (Angstroms) } & \multirow[b]{2}{*}{$\mathrm{Z}$} \\
\hline & $\mathrm{X}$ & $\mathrm{Y}$ & & \\
\hline 6 & -0.755022 & -0.840274 & 1.872335 & \\
\hline 6 & -0.761380 & -1.761549 & 0.846486 & \\
\hline 6 & -1.347079 & -1.603512 & -0.424776 & \\
\hline 6 & -1.878514 & -0.496226 & -1.089551 & \\
\hline 6 & -1.898165 & 0.904686 & -0.902690 & \\
\hline 6 & -1.280272 & 1.836115 & -0.061555 & \\
\hline 6 & -0.229050 & 1.740213 & 0.859619 & \\
\hline 6 & 0.728477 & 0.750603 & 1.017598 & \\
\hline 35 & 1.527743 & -0.101576 & -0.491856 & \\
\hline 1 & -1.503312 & -0.062215 & 1.909287 & \\
\hline 1 & -0.293542 & -1.098368 & 2.822041 & \\
\hline 1 & -0.208530 & -2.688393 & 0.994793 & \\
\hline 1 & -1.406403 & -2.524354 & -1.005186 & \\
\hline 1 & -2.418711 & -0.780954 & -1.993208 & \\
\hline 1 & -2.602778 & 1.382583 & -1.584740 & \\
\hline 1 & -1.740777 & 2.823322 & -0.098772 & \\
\hline 1 & -0.213360 & 2.508204 & 1.636303 & \\
\hline 1 & 1.442436 & 0.814996 & 1.829631 & \\
\hline \multicolumn{5}{|l|}{ out-TSf } \\
\hline Atomic & \multicolumn{3}{|c|}{ Coordinates (Angstroms) } & \\
\hline Number & $\mathrm{X}$ & $\mathrm{Y}$ & & $\mathrm{Z}$ \\
\hline 6 & 0.302591 & -1.287411 & 1.060265 & \\
\hline 6 & -0.615566 & -1.852089 & 0.195647 & \\
\hline
\end{tabular}




\begin{tabular}{|c|c|c|c|c|}
\hline 6 & -1.727314 & -1.202197 & -0.373853 & \\
\hline 6 & -2.111292 & 0.139511 & -0.390122 & \\
\hline 6 & -1.542625 & 1.385448 & -0.020977 & \\
\hline 6 & -0.274533 & 1.881859 & 0.288690 & \\
\hline 6 & 1.014790 & 1.325491 & 0.203490 & \\
\hline 6 & 1.372501 & 0.135004 & -0.410438 & \\
\hline 6 & 2.809999 & -0.301866 & -0.526303 & \\
\hline 1 & 0.027390 & -0.424697 & 1.649311 & \\
\hline 1 & 1.110877 & -1.894701 & 1.459988 & \\
\hline 1 & -0.439118 & -2.874603 & -0.139429 & \\
\hline 1 & -2.422818 & -1.863164 & -0.891209 & \\
\hline 1 & -3.126342 & 0.269349 & -0.768953 & \\
\hline 1 & -2.301884 & 2.168500 & 0.007021 & \\
\hline 1 & -0.291551 & 2.905098 & 0.664364 & \\
\hline 1 & 1.802151 & 1.872896 & 0.725016 & \\
\hline 1 & 0.699222 & -0.258616 & -1.161446 & \\
\hline 1 & 2.899749 & -1.392899 & -0.557730 & \\
\hline 1 & 3.257165 & 0.082042 & -1.454917 & \\
\hline 1 & 3.413853 & 0.068291 & 0.309602 & \\
\hline \multicolumn{5}{|l|}{ in-TSf } \\
\hline Atomic & \multicolumn{4}{|c|}{ Coordinates (Angstroms) } \\
\hline Number & $\mathrm{X}$ & $\mathrm{Y}$ & & $\mathrm{z}$ \\
\hline 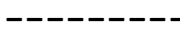 & -------- & -ーー-ー-ー- & -ーーーーーーーー & \\
\hline 6 & 0.200524 & -1.326113 & -1.287491 & \\
\hline 6 & 1.247756 & -1.394016 & -0.393700 & \\
\hline 6 & 1.911865 & -0.308085 & 0.217029 & \\
\hline 6 & 1.591899 & 1.043736 & 0.323675 & \\
\hline 6 & 0.464815 & 1.861025 & 0.047713 & \\
\hline 6 & -0.871294 & 1.681674 & -0.312632 & \\
\hline 6 & -1.708497 & 0.553630 & -0.435114 & \\
\hline 6 & -1.580135 & -0.712578 & 0.116713 & \\
\hline 6 & -1.012211 & -0.995802 & 1.480537 & \\
\hline 1 & 0.027280 & -0.422359 & -1.852432 & \\
\hline 1 & -0.191063 & -2.240163 & -1.726412 & \\
\hline 1 & 1.586058 & -2.383692 & -0.085520 & \\
\hline 1 & 2.852645 & -0.573756 & 0.700326 & \\
\hline 1 & 2.416849 & 1.632474 & 0.727177 & \\
\hline 1 & 0.723689 & 2.918272 & 0.122439 & \\
\hline 1 & -1.351544 & 2.609999 & -0.625461 & \\
\hline 1 & -2.546346 & 0.690897 & -1.121793 & \\
\hline 1 & -2.318392 & -1.443076 & -0.215366 & \\
\hline 1 & -0.689984 & -2.037185 & 1.576877 & \\
\hline 1 & -0.173798 & -0.347017 & 1.734764 & \\
\hline 1 & -1.803734 & -0.825213 & 2.225012 & \\
\hline \multicolumn{5}{|l|}{ out-TSg } \\
\hline Atomic & \multicolumn{4}{|c|}{ Coordinates (Angstroms) } \\
\hline Number & $\mathrm{X}$ & $\mathrm{Y}$ & & $\mathrm{Z}$ \\
\hline 6 & 0.158091 & -1.384131 & 1.079612 & \\
\hline 6 & -0.774584 & -1.859273 & 0.180584 & \\
\hline 6 & -1.806084 & -1.109397 & -0.420899 & \\
\hline 6 & -2.106213 & 0.254195 & -0.418552 & \\
\hline 6 & -1.491388 & 1.457767 & 0.009720 & \\
\hline 6 & -0.207047 & 1.900459 & 0.330996 & \\
\hline
\end{tabular}




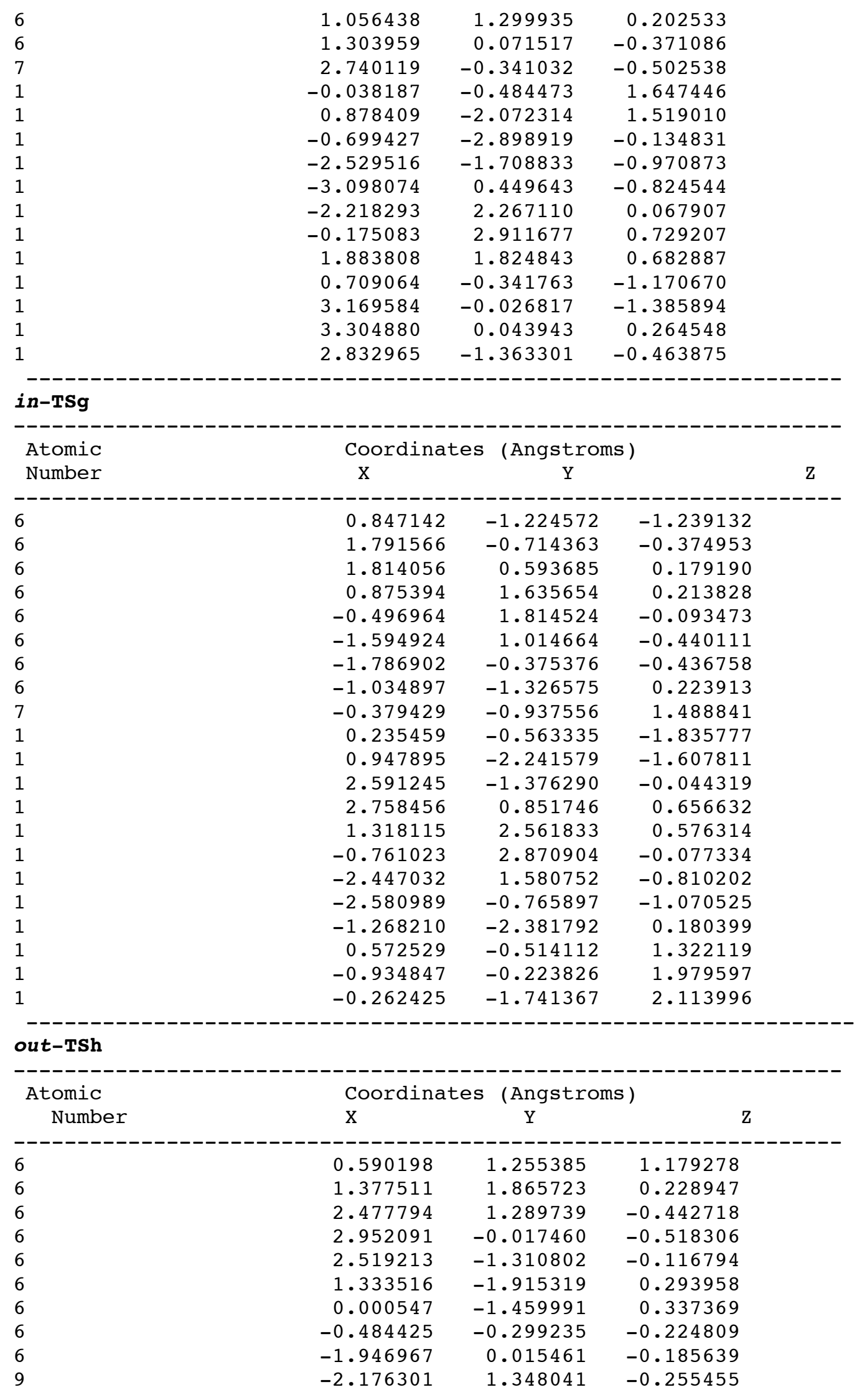




\begin{tabular}{|c|c|c|c|c|}
\hline 9 & -2.605958 & -0.540751 & -1.233705 & \\
\hline 9 & -2.540899 & -0.439865 & 0.940658 & \\
\hline 1 & 0.956585 & 0.396338 & 1.723011 & \\
\hline 1 & -0.230354 & 1.799432 & 1.637318 & \\
\hline 1 & 1.102093 & 2.870924 & -0.089015 & \\
\hline 1 & 3.077428 & 2.001577 & -1.009836 & \\
\hline 1 & 3.931260 & -0.067734 & -0.996096 & \\
\hline 1 & 3.340951 & -2.026472 & -0.165633 & \\
\hline 1 & 1.464168 & -2.938108 & 0.645197 & \\
\hline 1 & -0.692833 & -2.060426 & 0.923403 & \\
\hline 1 & 0.042252 & 0.176637 & -1.039544 & \\
\hline \multicolumn{5}{|l|}{ in-TSh } \\
\hline Atomic & \multirow{2}{*}{\multicolumn{3}{|c|}{$\mathrm{X}_{\mathrm{Y}}^{\text {Coordinates (Angstroms) }}$}} & \\
\hline Number & & & & $\mathrm{Z}$ \\
\hline 6 & -0.582728 & -1.122902 & 1.683114 & \\
\hline 6 & -0.886290 & -1.817736 & 0.540849 & \\
\hline 6 & -1.773771 & -1.418582 & -0.489241 & \\
\hline 6 & -2.286071 & -0.184668 & -0.852167 & \\
\hline 6 & -2.052102 & 1.174907 & -0.500052 & \\
\hline 6 & -1.157997 & 1.913792 & 0.265881 & \\
\hline 6 & 0.013303 & 1.630513 & 1.009601 & \\
\hline 6 & 0.941110 & 0.611838 & 0.951491 & \\
\hline 6 & 1.500880 & -0.005980 & -0.294720 & \\
\hline 9 & 1.925758 & -1.278652 & -0.102439 & \\
\hline 9 & 0.669498 & 0.000585 & -1.346618 & \\
\hline 9 & 2.608797 & 0.697895 & -0.662771 & \\
\hline 1 & -1.208097 & -0.306192 & 2.014029 & \\
\hline 1 & 0.064610 & -1.567152 & 2.433553 & \\
\hline 1 & -0.393638 & -2.776786 & 0.387972 & \\
\hline 1 & -2.103683 & -2.235895 & -1.131063 & \\
\hline 1 & -3.058574 & -0.262727 & -1.617518 & \\
\hline 1 & -2.813802 & 1.818407 & -0.942226 & \\
\hline 1 & -1.482163 & 2.946943 & 0.395437 & \\
\hline 1 & 0.163419 & 2.318208 & 1.844151 & \\
\hline 1 & 1.697457 & 0.599647 & 1.733589 & \\
\hline \multicolumn{5}{|l|}{ out-Tsi } \\
\hline Atomic & \multicolumn{3}{|c|}{ Coordinates (Angstroms) } & \\
\hline Number & $\mathrm{x}$ & $\mathrm{Y}$ & & $\mathrm{Z}$ \\
\hline 6 & 0.175594 & -1.245153 & 1.072283 & \\
\hline 6 & -0.682022 & -1.895107 & 0.200513 & \\
\hline 6 & -1.834792 & -1.347698 & -0.384324 & \\
\hline 6 & -2.343678 & -0.043644 & -0.419238 & \\
\hline 6 & -1.907308 & 1.253404 & -0.065083 & \\
\hline 6 & -0.703112 & 1.887325 & 0.272559 & \\
\hline 6 & 0.630497 & 1.470192 & 0.227676 & \\
\hline 6 & 1.099885 & 0.285862 & -0.346593 & \\
\hline 6 & 2.501200 & -0.000680 & -0.366475 & \\
\hline 7 & 3.637091 & -0.258396 & -0.370345 & \\
\hline 1 & -0.181592 & -0.408318 & 1.656035 & \\
\hline 1 & 1.036938 & -1.770705 & 1.474647 & \\
\hline 1 & -0.414478 & -2.901214 & -0.120545 & \\
\hline 1 & -2.460080 & -2.069163 & -0.909639 & \\
\hline
\end{tabular}




\begin{tabular}{|c|c|c|c|c|}
\hline 1 & -3.360792 & -0.018219 & -0.811683 & \\
\hline 1 & -2.739229 & 1.958528 & -0.074108 & \\
\hline 1 & -0.837048 & 2.907501 & 0.629523 & \\
\hline 1 & 1.356942 & 2.102652 & 0.733994 & \\
\hline 1 & 0.522113 & -0.179292 & -1.133721 & \\
\hline \multicolumn{5}{|l|}{ in-Tsi } \\
\hline Atomic & \multirow{2}{*}{\multicolumn{4}{|c|}{ Coordinates (Angstroms) }} \\
\hline Number & $\mathrm{x}$ & $\mathrm{Y}$ & & \\
\hline-- & ---- & -------1 & ---------- & \\
\hline 6 & 0.730876 & -0.596911 & -1.680078 & \\
\hline 6 & 1.734428 & -0.163048 & -0.831143 & \\
\hline 6 & 1.671658 & 0.936629 & 0.037469 & \\
\hline 6 & 0.615257 & 1.764445 & 0.438623 & \\
\hline 6 & -0.790007 & 1.770861 & 0.331736 & \\
\hline 6 & -1.808708 & 0.898964 & -0.089379 & \\
\hline 6 & -1.831014 & -0.453532 & -0.429588 & \\
\hline 6 & -0.843316 & -1.428047 & -0.217953 & \\
\hline 6 & -0.055783 & -1.445989 & 0.970070 & \\
\hline 7 & 0.576634 & -1.478192 & 1.947615 & \\
\hline 1 & -0.030694 & 0.093534 & -2.012994 & \\
\hline 1 & 0.911646 & -1.449162 & -2.329794 & \\
\hline 1 & 2.640721 & -0.763106 & -0.767588 & \\
\hline 1 & 2.625079 & 1.206259 & 0.489902 & \\
\hline 1 & 0.970135 & 2.637908 & 0.985841 & \\
\hline 1 & -1.199951 & 2.727437 & 0.657961 & \\
\hline 1 & -2.773979 & 1.393246 & -0.196588 & \\
\hline 1 & -2.696816 & -0.782246 & -1.003694 & \\
\hline 1 & -1.022915 & -2.416760 & -0.634901 & \\
\hline
\end{tabular}

\section{out-TSj}

\begin{tabular}{|c|c|c|c|c|}
\hline Atomic & \multicolumn{4}{|c|}{ Coordinates (Angstroms) } \\
\hline Number & $\mathrm{x}$ & $\mathrm{Y}$ & & $\mathrm{Z}$ \\
\hline 6 & -0.202515 & -1.162879 & 1.230636 & \\
\hline 6 & -1.046099 & -1.855582 & 0.389047 & \\
\hline 6 & -2.183484 & -1.337190 & -0.261868 & \\
\hline 6 & -2.668371 & -0.038234 & -0.422583 & \\
\hline 6 & -2.215590 & 1.282013 & -0.174932 & \\
\hline 6 & -1.007113 & 1.924663 & 0.109546 & \\
\hline 6 & 0.321913 & 1.478577 & 0.125792 & \\
\hline 6 & 0.772105 & 0.256414 & -0.332643 & \\
\hline 7 & 2.195113 & -0.041561 & -0.261981 & \\
\hline 8 & 2.603702 & -0.936990 & -1.006876 & \\
\hline 8 & 2.894618 & 0.582169 & 0.541348 & \\
\hline 1 & -0.537411 & -0.260316 & 1.722608 & \\
\hline 1 & 0.647467 & -1.665549 & 1.683863 & \\
\hline 1 & -0.789864 & -2.886343 & 0.147903 & \\
\hline 1 & -2.813292 & -2.093005 & -0.730366 & \\
\hline 1 & -3.676742 & -0.030966 & -0.837192 & \\
\hline 1 & -3.038351 & 1.993789 & -0.249716 & \\
\hline 1 & -1.123592 & 2.977045 & 0.362946 & \\
\hline 1 & 1.066038 & 2.123151 & 0.586637 & \\
\hline 1 & 0.288317 & -0.315004 & -1.106556 & \\
\hline
\end{tabular}

\section{$i n-T S j$}




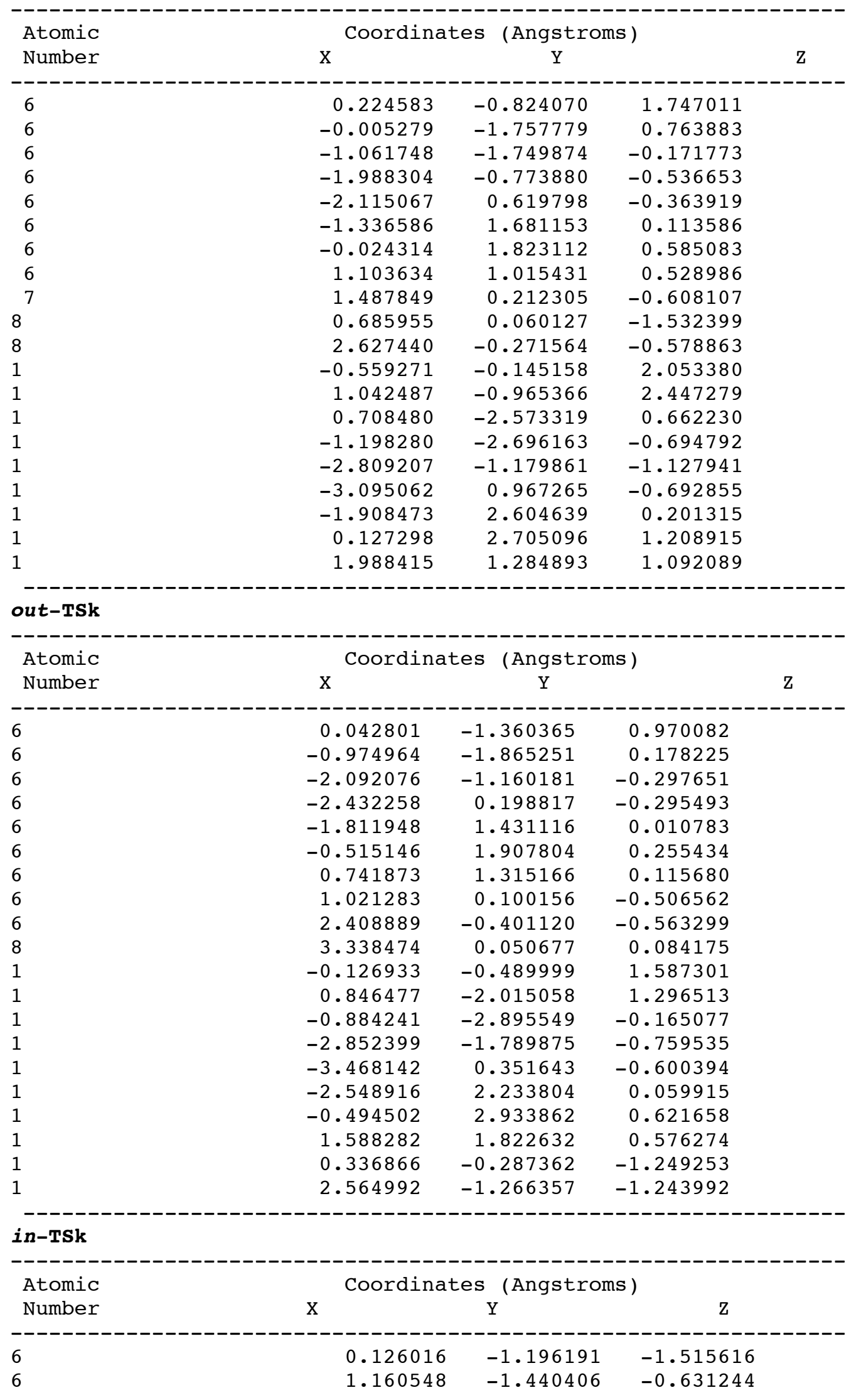




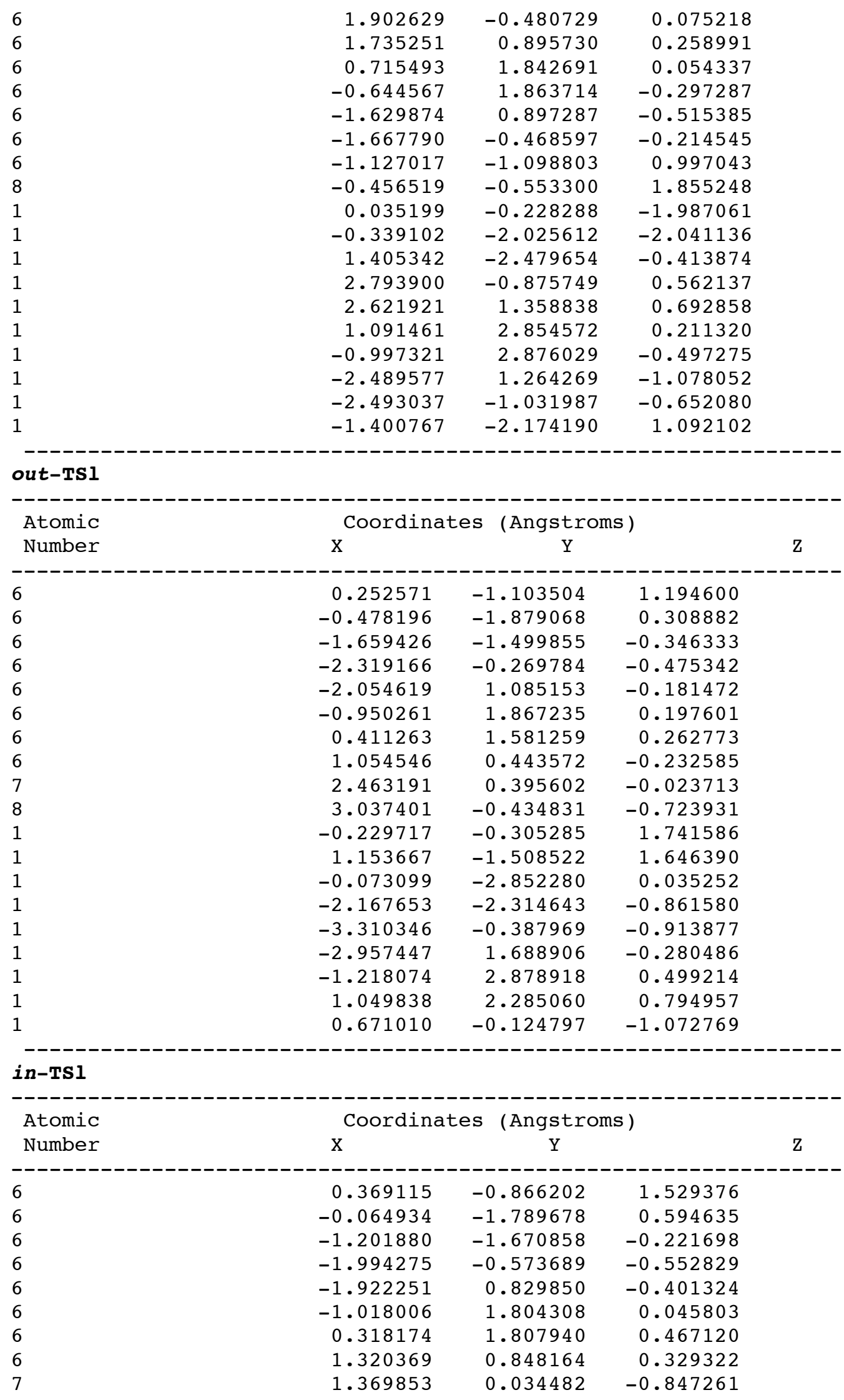




$\begin{array}{rrrr}8 & 2.497304 & -0.373610 & -1.097097 \\ 1 & -0.319206 & -0.128342 & 1.917345 \\ 1 & 1.225843 & -1.098013 & 2.155730 \\ 1 & 0.532489 & -2.689166 & 0.455026 \\ 1 & -1.516250 & -2.599244 & -0.698190 \\ 1 & -2.898230 & -0.863837 & -1.088518 \\ 1 & -2.857292 & 1.292872 & -0.719109 \\ 1 & -1.481694 & 2.787892 & 0.119983 \\ 1 & 0.616471 & 2.695420 & 1.025260 \\ 1 & 2.292592 & 1.010921 & 0.797641\end{array}$

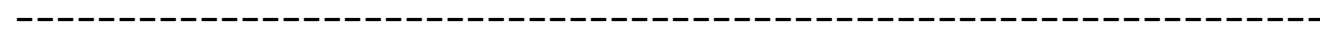

in, in-TSm

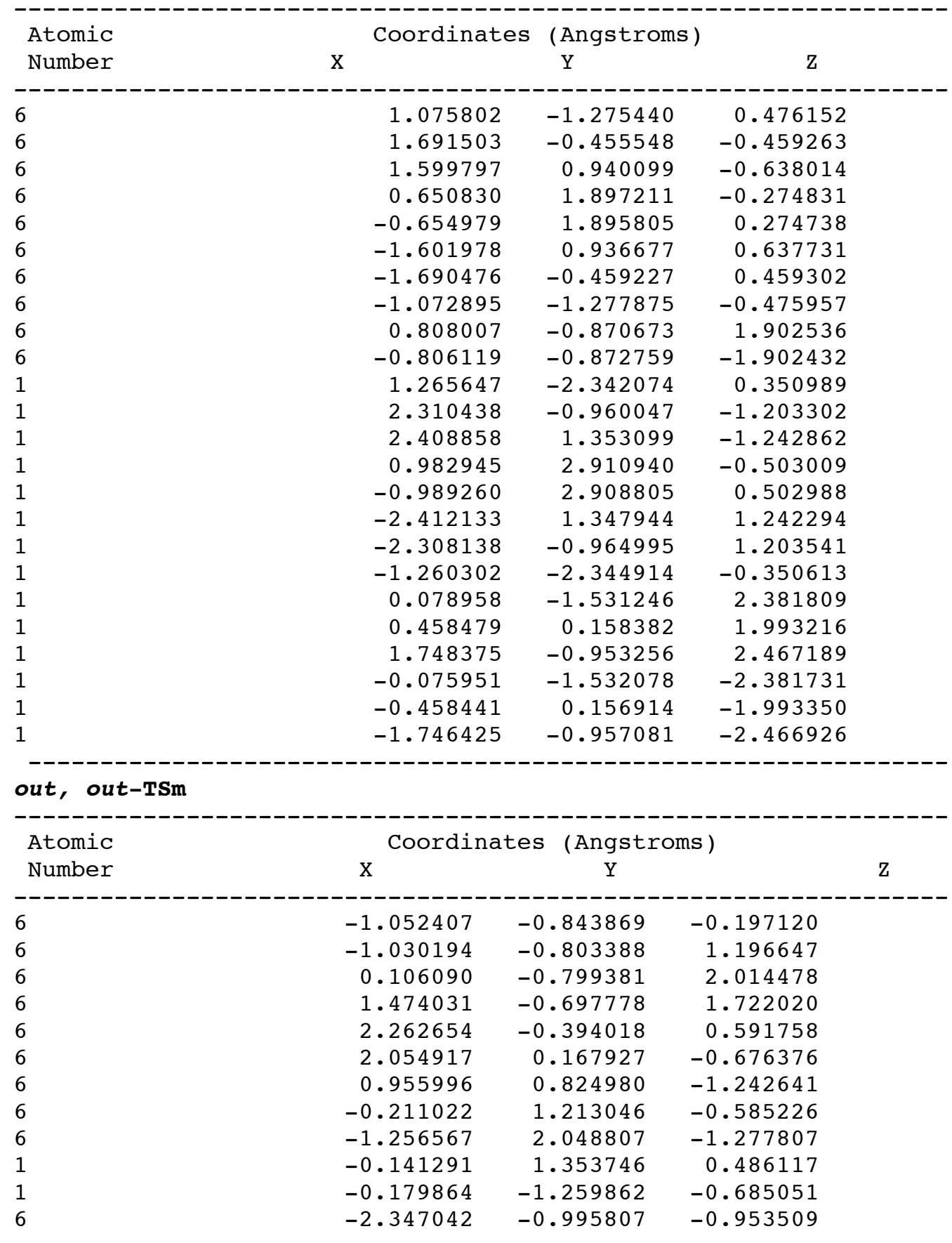




\begin{tabular}{|c|c|c|c|}
\hline 1 & 1.002818 & 0.994279 & -2.320082 \\
\hline 1 & 2.920001 & 0.087319 & -1.334659 \\
\hline 1 & 3.310898 & -0.646477 & 0.759042 \\
\hline 1 & 2.094631 & -0.932897 & 2.588107 \\
\hline 1 & -0.104006 & -0.910128 & 3.078333 \\
\hline 1 & -1.989078 & -0.706371 & 1.709354 \\
\hline 1 & -2.252328 & 1.894873 & -0.848280 \\
\hline 1 & -1.025329 & 3.118514 & -1.172070 \\
\hline 1 & -1.309189 & 1.828170 & -2.350051 \\
\hline 1 & -2.284013 & -0.568836 & -1.960213 \\
\hline 1 & -2.599570 & -2.059211 & -1.073180 \\
\hline 1 & -3.182417 & -0.516242 & -0.430711 \\
\hline \multicolumn{4}{|c|}{ out, in-TSm } \\
\hline \multicolumn{4}{|c|}{ Atomic Coordinates (Angstroms) } \\
\hline \multicolumn{4}{|l|}{ Number } \\
\hline 6 & -0.471207 & -1.427206 & 0.542474 \\
\hline 6 & 0.481713 & -1.763883 & -0.415312 \\
\hline 6 & 1.676598 & -1.109369 & -0.759955 \\
\hline 6 & 2.161865 & 0.185537 & -0.547403 \\
\hline 6 & 1.660317 & 1.407866 & -0.043057 \\
\hline 6 & 0.411922 & 1.906895 & 0.338546 \\
\hline 6 & -0.897194 & 1.418264 & 0.179565 \\
\hline 6 & -1.317383 & 0.375457 & -0.633484 \\
\hline 6 & -0.143695 & -0.855508 & 1.896361 \\
\hline 6 & -2.782509 & 0.074005 & -0.829586 \\
\hline 1 & -1.369027 & -2.046458 & 0.538658 \\
\hline 1 & 0.238426 & -2.618673 & -1.049783 \\
\hline 1 & 2.343455 & -1.715888 & -1.374411 \\
\hline 1 & 3.198397 & 0.288724 & -0.872128 \\
\hline 1 & 2.455415 & 2.143681 & 0.084654 \\
\hline 1 & 0.467561 & 2.863685 & 0.858824 \\
\hline 1 & -1.657996 & 1.906264 & 0.791837 \\
\hline 1 & -0.669160 & 0.098374 & -1.454683 \\
\hline 1 & -1.017689 & -0.385875 & 2.358931 \\
\hline 1 & 0.667172 & -0.127166 & 1.868292 \\
\hline 1 & 0.173250 & -1.680145 & 2.551571 \\
\hline 1 & -2.948425 & -0.970584 & -1.113847 \\
\hline 1 & -3.201789 & 0.694600 & -1.634921 \\
\hline 1 & -3.362147 & 0.277110 & 0.078112 \\
\hline \multicolumn{4}{|l|}{$4 a$} \\
\hline \multicolumn{4}{|c|}{ Atomic Coordinates (Angstroms) } \\
\hline Number & $\mathrm{x}$ & $\mathrm{Y}$ & $\mathrm{z}$ \\
\hline 6 & 0.752896 & -1.263365 & 0.660512 \\
\hline 6 & 1.833920 & -0.399392 & -0.011695 \\
\hline 6 & -0.363815 & -1.655476 & -0.266085 \\
\hline 6 & -1.498248 & -0.961062 & -0.451294 \\
\hline 6 & -1.825749 & 0.318141 & 0.169122 \\
\hline 6 & -1.035728 & 1.405104 & 0.325377 \\
\hline 6 & 0.311109 & 1.718966 & -0.167189 \\
\hline 6 & 1.446215 & 1.014711 & -0.346873 \\
\hline 1 & 0.348828 & -0.715710 & 1.519773 \\
\hline 1 & 1.231711 & -2.170446 & 1.050788 \\
\hline
\end{tabular}




\begin{tabular}{|c|c|c|c|c|}
\hline 1 & 2.720414 & -0.349303 & 0.641860 & \\
\hline 1 & 2.185690 & -0.901045 & -0.925086 & \\
\hline 1 & -0.240020 & -2.593124 & -0.807981 & \\
\hline 1 & -2.275751 & -1.396033 & -1.080862 & \\
\hline 1 & -2.859495 & 0.423476 & 0.501421 & \\
\hline 1 & -1.517910 & 2.266268 & 0.790377 & \\
\hline 1 & 0.399970 & 2.777951 & -0.417863 & \\
\hline 1 & 2.282960 & 1.592204 & -0.743678 & \\
\hline \multicolumn{5}{|l|}{$4 b$} \\
\hline \multicolumn{4}{|l|}{--------} & Coordinates (Angstroms) \\
\hline Number & $\mathrm{X}$ & $\mathrm{Y}$ & & $\mathrm{Z}$ \\
\hline \multicolumn{5}{|c|}{ 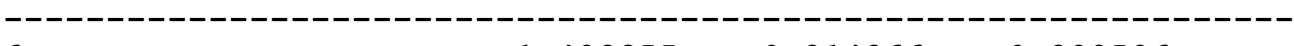 } \\
\hline 6 & -1.498855 & 0.314366 & 0.399596 & \\
\hline 6 & -0.665219 & -0.851027 & 0.972389 & \\
\hline 6 & -0.694430 & 1.483164 & -0.111101 & \\
\hline 6 & 0.628488 & 1.735434 & -0.166234 & \\
\hline 6 & 1.843220 & 0.976405 & 0.149357 & \\
\hline 6 & 2.167157 & -0.324876 & -0.033456 & \\
\hline 6 & 1.315841 & -1.417223 & -0.488865 & \\
\hline 6 & 0.058679 & -1.651503 & -0.076277 & \\
\hline 7 & -2.458927 & -0.070442 & -0.651690 & \\
\hline 1 & -2.116834 & 0.714605 & 1.220717 & \\
\hline 1 & 0.052446 & -0.453089 & 1.700682 & \\
\hline 1 & -1.348682 & -1.514662 & 1.520447 & \\
\hline 1 & -1.343414 & 2.291945 & -0.445826 & \\
\hline 1 & 0.873648 & 2.746987 & -0.496442 & \\
\hline 1 & 2.666779 & 1.620077 & 0.461613 & \\
\hline 1 & 3.213498 & -0.587573 & 0.128023 & \\
\hline 1 & 1.777551 & -2.124650 & -1.178970 & \\
\hline 1 & -0.467224 & -2.509087 & -0.494184 & \\
\hline 1 & -3.080304 & -0.797834 & -0.296324 & \\
\hline 1 & -1.944271 & -0.482072 & -1.430359 & \\
\hline \multicolumn{5}{|l|}{$4 \mathrm{C}$} \\
\hline \multicolumn{5}{|c|}{ Coordinates (Angstroms) } \\
\hline Number & $\mathrm{x}$ & $\mathrm{Y}$ & & $\mathrm{Z}$ \\
\hline \multicolumn{5}{|c|}{ 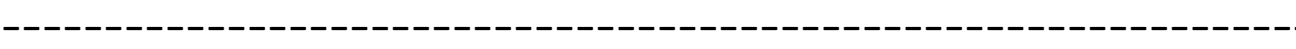 } \\
\hline 6 & -1.179278 & -0.093918 & -0.338101 & \\
\hline 6 & -1.013842 & 1.307451 & 0.240978 & \\
\hline 6 & -0.711387 & -1.190836 & 0.569275 & \\
\hline 6 & 0.521176 & -1.718277 & 0.568381 & \\
\hline 6 & 1.628992 & -1.272183 & -0.270225 & \\
\hline 6 & 2.019349 & -0.003275 & -0.527647 & \\
\hline 6 & 1.587257 & 1.281525 & 0.038926 & \\
\hline 6 & 0.396736 & 1.806868 & 0.385961 & \\
\hline 9 & -2.554063 & -0.283067 & -0.562542 & \\
\hline 1 & -0.676952 & -0.156624 & -1.310678 & \\
\hline 1 & -1.519416 & 1.341044 & 1.216147 & \\
\hline 1 & -1.567838 & 2.010244 & -0.400572 & \\
\hline 1 & -1.474316 & -1.579837 & 1.241256 & \\
\hline 1 & 0.718652 & -2.577425 & 1.209931 & \\
\hline 1 & 2.240299 & -2.066233 & -0.700409 & \\
\hline 1 & 2.900426 & 0.095308 & -1.163262 & \\
\hline 1 & 2.433221 & 1.957555 & 0.176861 & \\
\hline 1 & 0.438478 & 2.819447 & 0.788315 & \\
\hline
\end{tabular}




\section{$4 d$}

\begin{tabular}{|c|c|c|c|c|}
\hline Atomic & \multicolumn{4}{|c|}{ Coordinates (Angstroms) } \\
\hline Number & $\mathrm{X}$ & $\mathrm{Y}$ & & $\mathrm{Z}$ \\
\hline 6 & -0.816382 & 0.023940 & -0.144381 & \\
\hline 6 & -0.508918 & 1.430046 & 0.365620 & \\
\hline 6 & -0.357544 & -1.068575 & 0.772590 & \\
\hline 6 & 0.813411 & -1.709710 & 0.636532 & \\
\hline 6 & 1.838291 & -1.391232 & -0.352047 & \\
\hline 6 & 2.303933 & -0.164706 & -0.680917 & \\
\hline 6 & 2.051507 & 1.154694 & -0.084720 & \\
\hline 6 & 0.955755 & 1.786711 & 0.377363 & \\
\hline 17 & -2.637152 & -0.130445 & -0.392154 & \\
\hline 1 & -0.389732 & -0.108572 & -1.139966 & \\
\hline 1 & -1.034717 & 2.168943 & -0.256610 & \\
\hline 1 & -0.924054 & 1.546598 & 1.375509 & \\
\hline 1 & -1.043713 & -1.351352 & 1.567290 & \\
\hline 1 & 1.021707 & -2.551805 & 1.297009 & \\
\hline 1 & 2.317844 & -2.244331 & -0.832578 & \\
\hline 1 & 3.108569 & -0.147675 & -1.417162 & \\
\hline 1 & 2.965704 & 1.749407 & -0.036595 & \\
\hline 1 & 1.129653 & 2.789349 & 0.769479 & \\
\hline
\end{tabular}

$4 e$

\begin{tabular}{|c|c|c|c|c|}
\hline \multirow{2}{*}{$\begin{array}{l}\text { Atomic } \\
\text { Number }\end{array}$} & \multicolumn{4}{|c|}{ Coordinates (Angstroms) } \\
\hline & $\mathrm{X}$ & $\mathrm{Y}$ & & $\mathrm{Z}$ \\
\hline 6 & -0.816382 & 0.023940 & -0.144381 & \\
\hline 6 & -0.508918 & 1.430046 & 0.365620 & \\
\hline 6 & -0.357544 & -1.068575 & 0.772590 & \\
\hline 6 & 0.813411 & -1.709710 & 0.636532 & \\
\hline 6 & 1.838291 & -1.391232 & -0.352047 & \\
\hline 6 & 2.303933 & -0.164706 & -0.680917 & \\
\hline 6 & 2.051507 & 1.154694 & -0.084720 & \\
\hline 6 & 0.955755 & 1.786711 & 0.377363 & \\
\hline 17 & -2.637152 & -0.130445 & -0.392154 & \\
\hline 1 & -0.389732 & -0.108572 & -1.139966 & \\
\hline 1 & -1.034717 & 2.168943 & -0.256610 & \\
\hline 1 & -0.924054 & 1.546598 & 1.375509 & \\
\hline 1 & -1.043713 & -1.351352 & 1.567290 & \\
\hline 1 & 1.021707 & -2.551805 & 1.297009 & \\
\hline 1 & 2.317844 & -2.244331 & -0.832578 & \\
\hline 1 & 3.108569 & -0.147675 & -1.417162 & \\
\hline 1 & 2.965704 & 1.749407 & -0.036595 & \\
\hline 1 & 1.129653 & 2.789349 & 0.769479 & \\
\hline \multicolumn{5}{|l|}{$4 f$} \\
\hline \multicolumn{4}{|l|}{--------} & Coordinates (Angstroms) \\
\hline Number & $\mathrm{X}$ & $\mathrm{Y}$ & & $\mathrm{Z}$ \\
\hline 6 & 1.462072 & -0.234714 & -0.319074 & \\
\hline 6 & 0.528569 & -1.184368 & 0.459289 & \\
\hline 6 & 0.869162 & 1.121436 & -0.617562 & \\
\hline 6 & -0.282699 & 1.726280 & -0.263952 & \\
\hline
\end{tabular}




$\begin{array}{rrrr}6 & -1.506311 & 1.306075 & 0.429414 \\ 6 & -2.219616 & 0.158656 & 0.365271 \\ 6 & -1.870854 & -1.073309 & -0.332391 \\ 6 & -0.660820 & -1.656113 & -0.328166 \\ 6 & 2.800253 & -0.043294 & 0.427563 \\ 1 & 1.698710 & -0.711304 & -1.283069 \\ 1 & 1.116219 & -2.057052 & 0.773348 \\ 1 & 0.196360 & -0.675042 & 1.371916 \\ 1 & 1.572009 & 1.765022 & -1.150609 \\ 1 & -0.331610 & 2.782610 & -0.537514 \\ 1 & -1.979212 & 2.122854 & 0.976731 \\ 1 & -3.198663 & 0.167879 & 0.846179 \\ 1 & -2.684928 & -1.568688 & -0.863322 \\ 1 & -0.526529 & -2.566870 & -0.911907 \\ 1 & 3.471235 & 0.627334 & -0.121689 \\ 1 & 2.632192 & 0.391473 & 1.420024 \\ 1 & 3.315684 & -1.002110 & 0.557556\end{array}$

$4 \mathrm{~g}$

\begin{tabular}{|c|c|c|c|c|}
\hline \multirow{2}{*}{$\begin{array}{l}\text { Atomic } \\
\text { Number }\end{array}$} & \multicolumn{3}{|c|}{ Coordinates (Angstroms) } & \multirow[b]{2}{*}{$\mathrm{Z}$} \\
\hline & $\mathrm{x}$ & $\mathrm{Y}$ & & \\
\hline 6 & -1.329578 & -0.697937 & 0.028496 & \\
\hline 6 & -1.636952 & 0.728814 & -0.440821 & \\
\hline 6 & -0.348261 & -1.463273 & -0.822646 & \\
\hline 6 & 0.991849 & -1.422551 & -0.752825 & \\
\hline 6 & 1.830003 & -0.587179 & 0.108082 & \\
\hline 6 & 1.704990 & 0.738352 & 0.379962 & \\
\hline 6 & 0.766326 & 1.756613 & -0.103805 & \\
\hline 6 & -0.538490 & 1.761173 & -0.443321 & \\
\hline 7 & -0.851278 & -0.671491 & 1.487038 & \\
\hline 1 & -2.272979 & -1.249676 & 0.069462 & \\
\hline 1 & -2.030159 & 0.629446 & -1.460749 & \\
\hline 1 & -2.483855 & 1.128216 & 0.141383 & \\
\hline 1 & -0.814157 & -2.124370 & -1.548471 & \\
\hline 1 & 1.540032 & -2.117104 & -1.388354 & \\
\hline 1 & 2.722917 & -1.076521 & 0.494990 & \\
\hline 1 & 2.508104 & 1.161798 & 0.982141 & \\
\hline 1 & 1.239609 & 2.736243 & -0.153002 & \\
\hline 1 & -0.926668 & 2.733747 & -0.742073 & \\
\hline 1 & 0.124081 & -0.311126 & 1.517957 & \\
\hline 1 & -0.841683 & -1.617438 & 1.883502 & \\
\hline 1 & -1.445620 & -0.076845 & 2.075216 & \\
\hline \multicolumn{5}{|l|}{$4 \mathrm{~h}$} \\
\hline \multicolumn{5}{|c|}{ Coordinates (Angstroms) } \\
\hline Number & $\mathrm{x}$ & $\mathrm{Y}$ & & $\mathrm{z}$ \\
\hline 6 & 0.338000 & 0.049245 & -0.049549 & \\
\hline 6 & -0.057443 & 1.439246 & 0.486184 & \\
\hline 6 & -0.160013 & -1.071932 & 0.825815 & \\
\hline 6 & -1.326418 & -1.710283 & 0.654717 & \\
\hline 6 & -2.328545 & -1.400537 & -0.359863 & \\
\hline 6 & -2.799212 & -0.184868 & -0.718117 & \\
\hline 6 & -2.578123 & 1.149085 & -0.145323 & \\
\hline 6 & -1.514386 & 1.792768 & 0.372873 & \\
\hline
\end{tabular}




\begin{tabular}{|c|c|c|c|}
\hline 6 & 1.849832 & -0.042897 & -0.202210 \\
\hline 9 & 2.229907 & -1.246874 & -0.679566 \\
\hline 9 & 2.485588 & 0.136936 & 0.982423 \\
\hline 9 & 2.326207 & 0.893991 & -1.054229 \\
\hline 1 & -0.073220 & -0.066059 & -1.058636 \\
\hline 1 & 0.258537 & 1.520484 & 1.534765 \\
\hline 1 & 0.512607 & 2.213237 & -0.048843 \\
\hline 1 & 0.500714 & -1.382016 & 1.632968 \\
\hline 1 & -1.543314 & -2.564141 & 1.297298 \\
\hline 1 & -2.786592 & -2.263178 & -0.844683 \\
\hline 1 & -3.581506 & -0.190319 & -1.478104 \\
\hline 1 & -3.489703 & 1.748624 & -0.176909 \\
\hline 1 & -1.714995 & 2.807927 & 0.717321 \\
\hline
\end{tabular}

\section{$4 i$}

\begin{tabular}{|c|c|c|c|c|}
\hline \multirow{2}{*}{$\begin{array}{l}\text { Atomic } \\
\text { Number }\end{array}$} & \multicolumn{3}{|c|}{ Coordinates (Angstroms) } & \multirow[b]{2}{*}{$\mathrm{Z}$} \\
\hline & $\mathrm{x}$ & $\mathrm{Y}$ & & \\
\hline 6 & -0.944014 & 0.012155 & -0.165800 & \\
\hline 6 & -0.602246 & 1.419272 & 0.384789 & \\
\hline 6 & -0.469325 & -1.104628 & 0.739978 & \\
\hline 6 & 0.715049 & -1.720763 & 0.616655 & \\
\hline 6 & 1.753052 & -1.389060 & -0.353871 & \\
\hline 6 & 2.218740 & -0.162821 & -0.681337 & \\
\hline 6 & 1.953194 & 1.161082 & -0.103180 & \\
\hline 6 & 0.855498 & 1.787251 & 0.362198 & \\
\hline 6 & -2.396815 & -0.095024 & -0.382545 & \\
\hline 7 & -3.544626 & -0.173244 & -0.538663 & \\
\hline 1 & -0.458398 & -0.093428 & -1.144073 & \\
\hline 1 & -1.150470 & 2.178396 & -0.193518 & \\
\hline 1 & -0.988760 & 1.501709 & 1.409229 & \\
\hline 1 & -1.165627 & -1.427375 & 1.510697 & \\
\hline 1 & 0.918477 & -2.572183 & 1.266551 & \\
\hline 1 & 2.243533 & -2.240636 & -0.825900 & \\
\hline 1 & 3.032446 & -0.148120 & -1.407293 & \\
\hline 1 & 2.858938 & 1.769433 & -0.076031 & \\
\hline 1 & 1.023446 & 2.800129 & 0.729644 & \\
\hline \multicolumn{5}{|l|}{$4 j$} \\
\hline \multicolumn{5}{|l|}{ Atomic } \\
\hline Number & $\mathrm{X}$ & $\mathrm{Y}$ & & $\mathrm{Z}$ \\
\hline \multicolumn{5}{|c|}{ 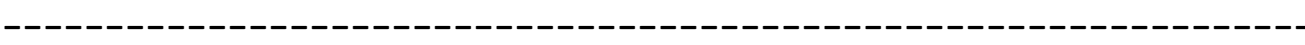 } \\
\hline 6 & 0.596609 & 0.012284 & 0.118367 & \\
\hline 6 & 0.285228 & 1.418062 & -0.414805 & \\
\hline 6 & 0.132278 & -1.092566 & -0.783247 & \\
\hline 6 & -1.044826 & -1.718886 & -0.641600 & \\
\hline 6 & -2.071383 & -1.385250 & 0.339343 & \\
\hline 6 & -2.527288 & -0.156661 & 0.674244 & \\
\hline 6 & -2.263979 & 1.168850 & 0.099288 & \\
\hline 6 & -1.169296 & 1.796484 & -0.371713 & \\
\hline 7 & 2.110488 & -0.077453 & 0.277672 & \\
\hline 8 & 2.784498 & -0.068153 & -0.750103 & \\
\hline 8 & 2.554414 & -0.123077 & 1.419480 & \\
\hline 1 & 0.208381 & -0.104463 & 1.130410 & \\
\hline 1 & 0.845186 & 2.163916 & 0.171330 & \\
\hline 1 & 0.667061 & 1.495845 & -1.440139 & \\
\hline
\end{tabular}




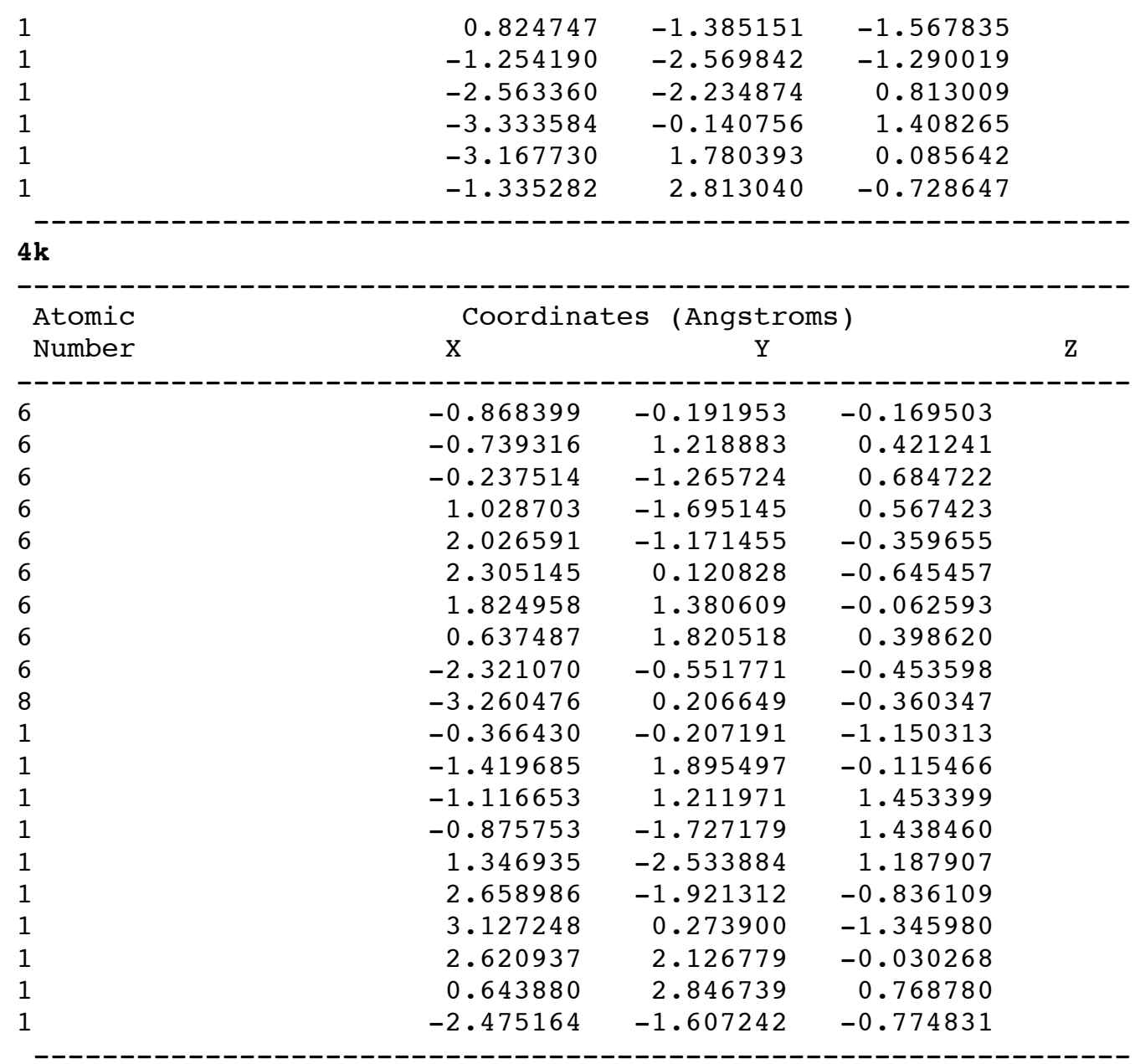

41

\begin{tabular}{|c|c|c|c|c|}
\hline Atomic & \multicolumn{3}{|c|}{ Coordinates (Angstroms) } & \\
\hline Number & $\mathrm{X}$ & $\mathrm{Y}$ & & $\mathrm{Z}$ \\
\hline 6 & 0.908924 & -1.017455 & -0.311003 & \\
\hline 6 & 1.442250 & 0.227978 & -1.026216 & \\
\hline 6 & -0.381575 & -1.567483 & -0.848692 & \\
\hline 6 & -1.608532 & -1.183768 & -0.468263 & \\
\hline 6 & -1.938558 & -0.125098 & 0.481246 & \\
\hline 6 & -1.443023 & 1.130724 & 0.553049 & \\
\hline 6 & -0.475653 & 1.855337 & -0.271313 & \\
\hline 6 & 0.661853 & 1.509119 & -0.905995 & \\
\hline 7 & 0.838806 & -0.876811 & 1.213207 & \\
\hline 8 & 1.547043 & 0.002230 & 1.641550 & \\
\hline 1 & 1.672175 & -1.807038 & -0.405204 & \\
\hline 1 & 2.464545 & 0.432594 & -0.678933 & \\
\hline 1 & 1.539488 & -0.027750 & -2.089890 & \\
\hline 1 & -0.291351 & -2.370007 & -1.578405 & \\
\hline 1 & -2.459443 & -1.738483 & -0.866209 & \\
\hline 1 & -2.745182 & -0.365634 & 1.173876 & \\
\hline 1 & -1.895204 & 1.763821 & 1.317124 & \\
\hline 1 & -0.706041 & 2.920656 & -0.318838 & \\
\hline 1 & 1.178909 & 2.335564 & -1.395239 & \\
\hline
\end{tabular}


trans $-4 m$

\begin{tabular}{|c|c|c|c|c|}
\hline \multirow{2}{*}{$\begin{array}{l}\text { Atomic } \\
\text { Number }\end{array}$} & \multicolumn{4}{|c|}{ Coordinates (Angstroms) } \\
\hline & $\mathrm{x}$ & $\mathrm{Y}$ & & $\mathrm{Z}$ \\
\hline 6 & -0.810384 & -0.677692 & -0.195347 & \\
\hline 6 & -0.760857 & -0.593895 & 1.310657 & \\
\hline 6 & 0.344611 & -0.661992 & 2.069598 & \\
\hline 6 & 1.711486 & -0.684735 & 1.563279 & \\
\hline 6 & 2.224388 & 0.108375 & 0.595805 & \\
\hline 6 & 1.633092 & 1.264829 & -0.086144 & \\
\hline 6 & 0.413974 & 1.551541 & -0.585127 & \\
\hline 6 & -0.831974 & 0.731177 & -0.839254 & \\
\hline 1 & 0.103328 & -1.177517 & -0.542564 & \\
\hline 6 & -2.018846 & -1.539125 & -0.609237 & \\
\hline 6 & -1.064810 & 0.693388 & -2.367494 & \\
\hline 1 & -1.681919 & 1.279720 & -0.401918 & \\
\hline 1 & -1.728749 & -0.543674 & 1.812561 & \\
\hline 1 & 0.230587 & -0.726493 & 3.152716 & \\
\hline 1 & 2.400830 & -1.366871 & 2.062296 & \\
\hline 1 & 3.287742 & -0.016920 & 0.386481 & \\
\hline 1 & 2.364441 & 2.067497 & -0.207065 & \\
\hline 1 & 0.326125 & 2.555059 & -1.006477 & \\
\hline 1 & -2.010366 & -2.491204 & -0.066951 & \\
\hline 1 & -2.965412 & -1.033187 & -0.376339 & \\
\hline 1 & -2.013700 & -1.767736 & -1.679203 & \\
\hline 1 & -1.000003 & 1.701299 & -2.793506 & \\
\hline 1 & -0.305164 & 0.075739 & -2.861810 & \\
\hline 1 & -2.051815 & 0.293058 & -2.618640 & \\
\hline \multicolumn{5}{|l|}{ cis-4m } \\
\hline \multicolumn{5}{|c|}{ Atomic Coordinates (Angstroms) } \\
\hline Number & $\mathrm{X}$ & $\mathrm{Y}$ & & $\mathrm{Z}$ \\
\hline \multicolumn{5}{|c|}{ 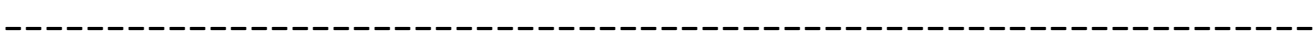 } \\
\hline 1 & -0.056336 & 0.307406 & -2.384700 & \\
\hline 6 & -0.174185 & 0.114151 & -1.309302 & \\
\hline 6 & 1.284930 & 0.130889 & -0.756902 & \\
\hline 6 & 2.220613 & -0.756121 & -1.609951 & \\
\hline 6 & -0.994901 & 1.272198 & -0.785896 & \\
\hline 6 & -1.657427 & 1.397201 & 0.377305 & \\
\hline 6 & -1.709131 & 0.488804 & 1.513650 & \\
\hline 6 & -0.723838 & -0.252480 & 2.071845 & \\
\hline 6 & 0.703028 & -0.375773 & 1.785101 & \\
\hline 6 & 1.491967 & -0.212272 & 0.700819 & \\
\hline 6 & -0.871957 & -1.255517 & -1.200430 & \\
\hline 1 & 1.641688 & 1.163822 & -0.890276 & \\
\hline 1 & -1.074839 & 2.115487 & -1.472721 & \\
\hline 1 & -2.271005 & 2.292451 & 0.494798 & \\
\hline 1 & -2.667412 & 0.470685 & 2.034275 & \\
\hline 1 & -1.015186 & -0.806328 & 2.964776 & \\
\hline 1 & 1.251279 & -0.700031 & 2.671272 & \\
\hline 1 & 2.547216 & -0.405266 & 0.904457 & \\
\hline 1 & 3.266701 & -0.613804 & -1.314949 & \\
\hline 1 & 1.987796 & -1.819920 & -1.492669 & \\
\hline 1 & 2.136670 & -0.503579 & -2.673335 & \\
\hline 1 & -0.291621 & -2.025536 & -1.719779 & \\
\hline 1 & -1.010361 & -1.573312 & -0.166078 & \\
\hline
\end{tabular}




\begin{tabular}{|c|c|c|c|}
\hline Atomic & \multicolumn{3}{|c|}{ Coordinates (Angstroms) } \\
\hline Number & $\mathrm{x}$ & $\mathrm{Y}$ & $\mathrm{Z}$ \\
\hline 6 & 0.282352 & -1.370281 & 0.584311 \\
\hline 6 & 1.362208 & -1.248898 & -0.280779 \\
\hline 6 & 1.965553 & -0.016043 & -0.613745 \\
\hline 6 & 1.618516 & 1.204314 & -0.032285 \\
\hline 6 & -0.759667 & -0.423122 & 0.704356 \\
\hline 6 & 0.351170 & 1.573585 & 0.440817 \\
\hline 6 & -1.611599 & -0.151498 & -0.466026 \\
\hline 6 & -2.902559 & 0.202545 & -0.406801 \\
\hline 1 & 0.304511 & -2.200971 & 1.292289 \\
\hline 1 & 1.967627 & -2.134830 & -0.469237 \\
\hline 1 & 2.952027 & -0.084774 & -1.070399 \\
\hline 1 & 2.463441 & 1.847880 & 0.227156 \\
\hline 1 & -1.310706 & -0.427691 & 1.647144 \\
\hline 1 & -0.482284 & 1.646125 & -0.238665 \\
\hline 1 & 0.310667 & 2.324317 & 1.233474 \\
\hline 1 & -1.134850 & -0.291077 & -1.435882 \\
\hline 1 & -3.489354 & 0.358217 & -1.307050 \\
\hline 1 & -3.416918 & 0.339193 & 0.542081 \\
\hline
\end{tabular}

\section{TS3a}

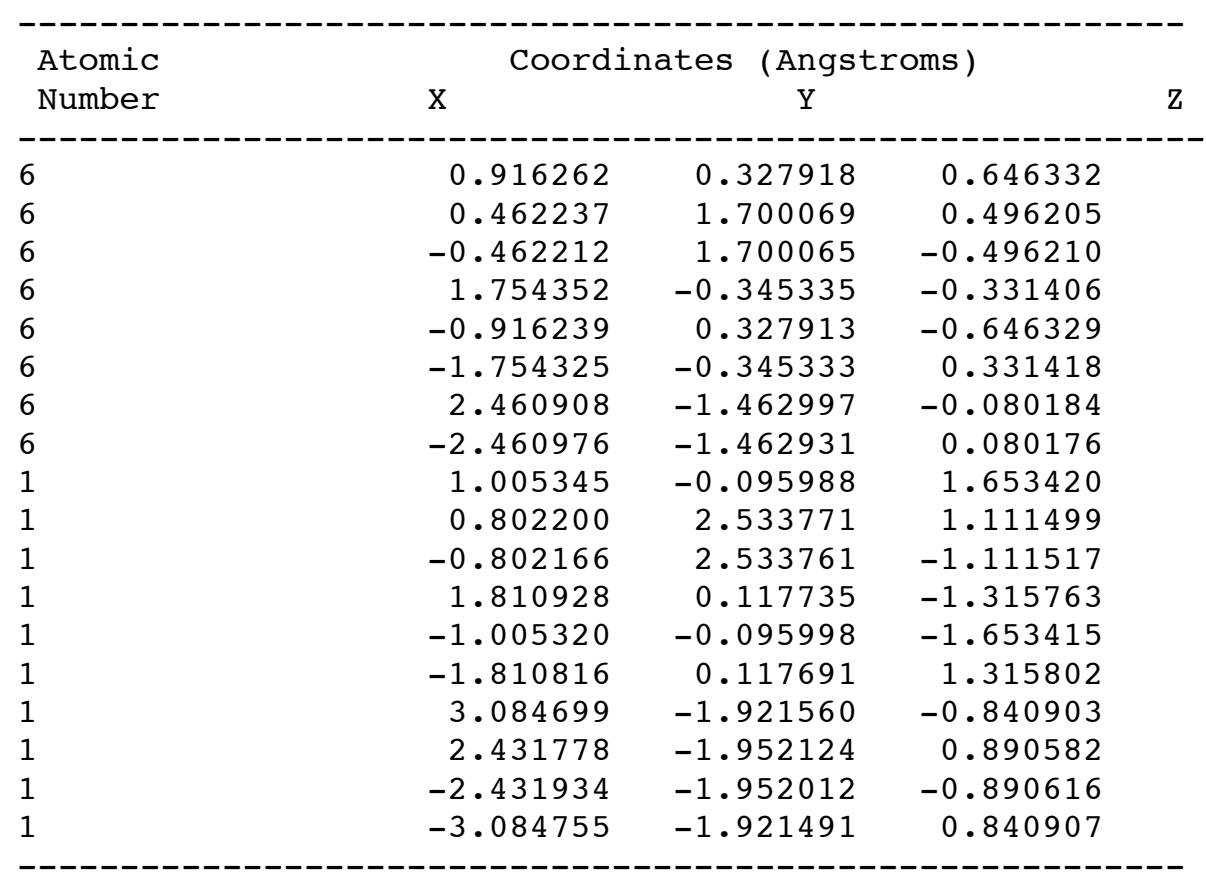

\section{Ts4a}

\begin{tabular}{|c|c|c|c|c|}
\hline Atomic & \multicolumn{4}{|c|}{ Coordinates (Angstroms) } \\
\hline Number & $\mathrm{x}$ & $\mathrm{Y}$ & & $\mathrm{Z}$ \\
\hline 6 & & & & \\
\hline $\begin{array}{l}6 \\
6\end{array}$ & $\begin{array}{l}-0.142691 \\
-2.088922\end{array}$ & 0.610205 & $\begin{array}{r}0.160350 \\
-0.187888\end{array}$ & \\
\hline 6 & -2.856368 & -0.108913 & -0.089083 & \\
\hline
\end{tabular}




\begin{tabular}{lrrr}
6 & 0.081280 & -0.212128 & -0.713262 \\
6 & -2.101443 & -1.004890 & 0.733950 \\
6 & 1.430353 & -0.364583 & -0.662948 \\
6 & 2.348576 & 0.213261 & 0.299816 \\
6 & 3.675452 & -0.002747 & 0.301299 \\
1 & -0.168912 & 1.255802 & 0.835168 \\
1 & -2.393370 & 2.042782 & -0.392146 \\
1 & -3.813029 & -0.330935 & -0.561684 \\
1 & -0.460352 & -0.728567 & -1.503400 \\
1 & -1.857805 & -0.706957 & 1.747279 \\
1 & -2.079433 & -2.086481 & 0.573970 \\
1 & 1.886541 & -0.995475 & -1.425060 \\
1 & 1.932655 & 0.860511 & 1.071014 \\
1 & 4.148309 & -0.644853 & -0.438830 \\
1 & 4.328012 & 0.452012 & 1.040287 \\
\hline
\end{tabular}
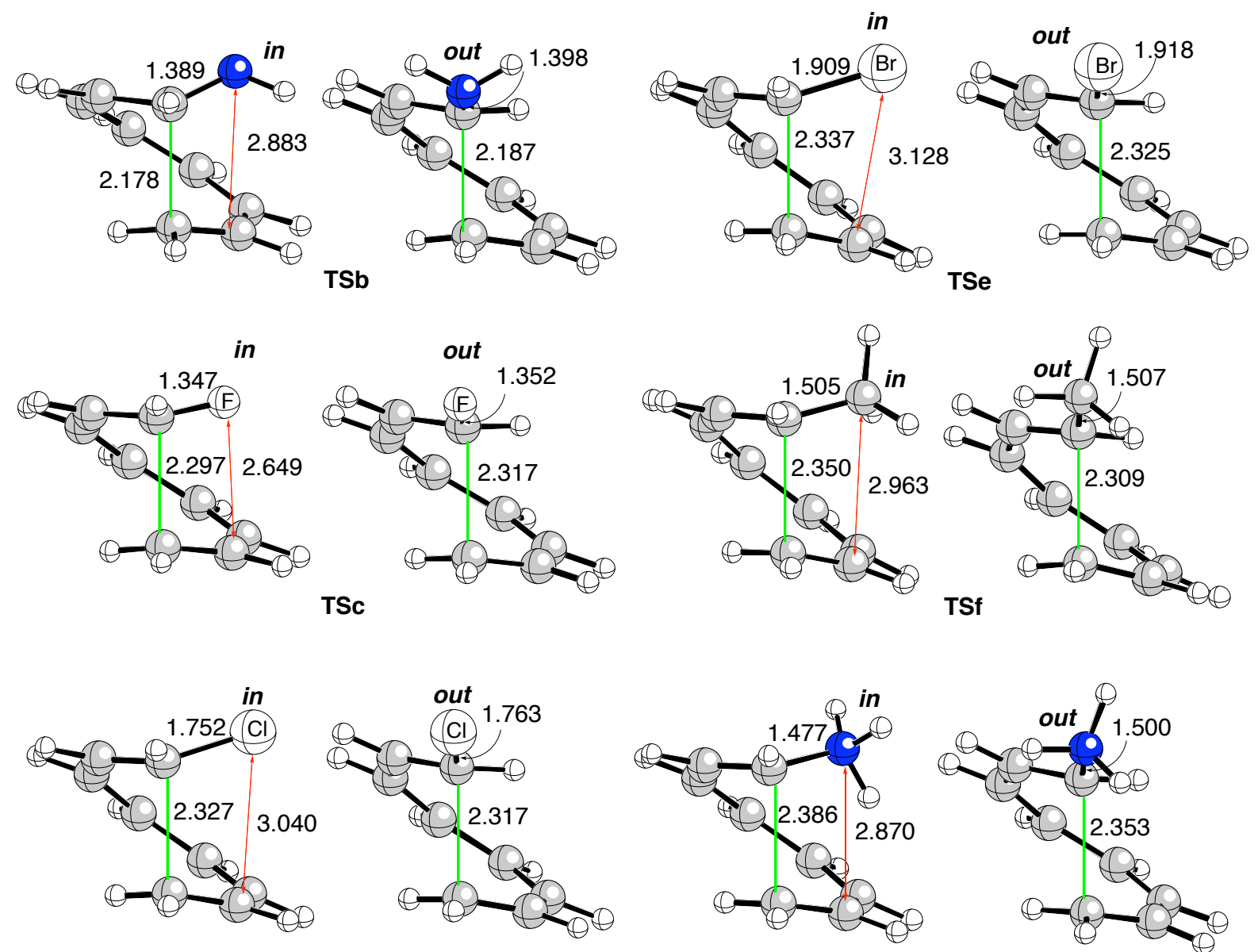

TSd
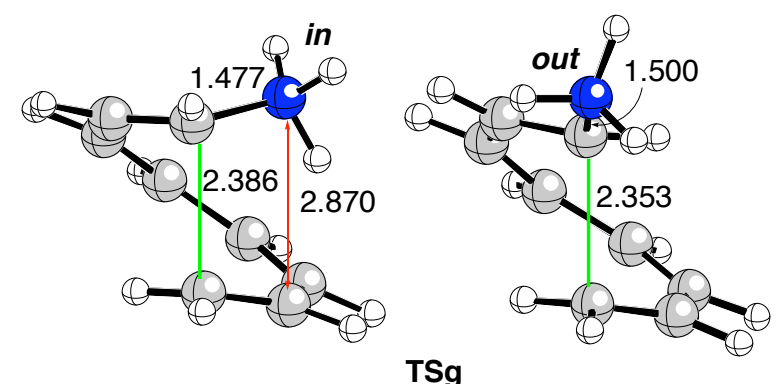

Figure S1. Chief geometric features (B3LYP/6-31G* level of theory) of transition structures in- and out-TSb-g (see Scheme 2). Bond distances are given in $\AA$. Unless otherwise stated, white, gray and blue colors denote hydrogen, carbon and nitrogen atoms, respectively. 

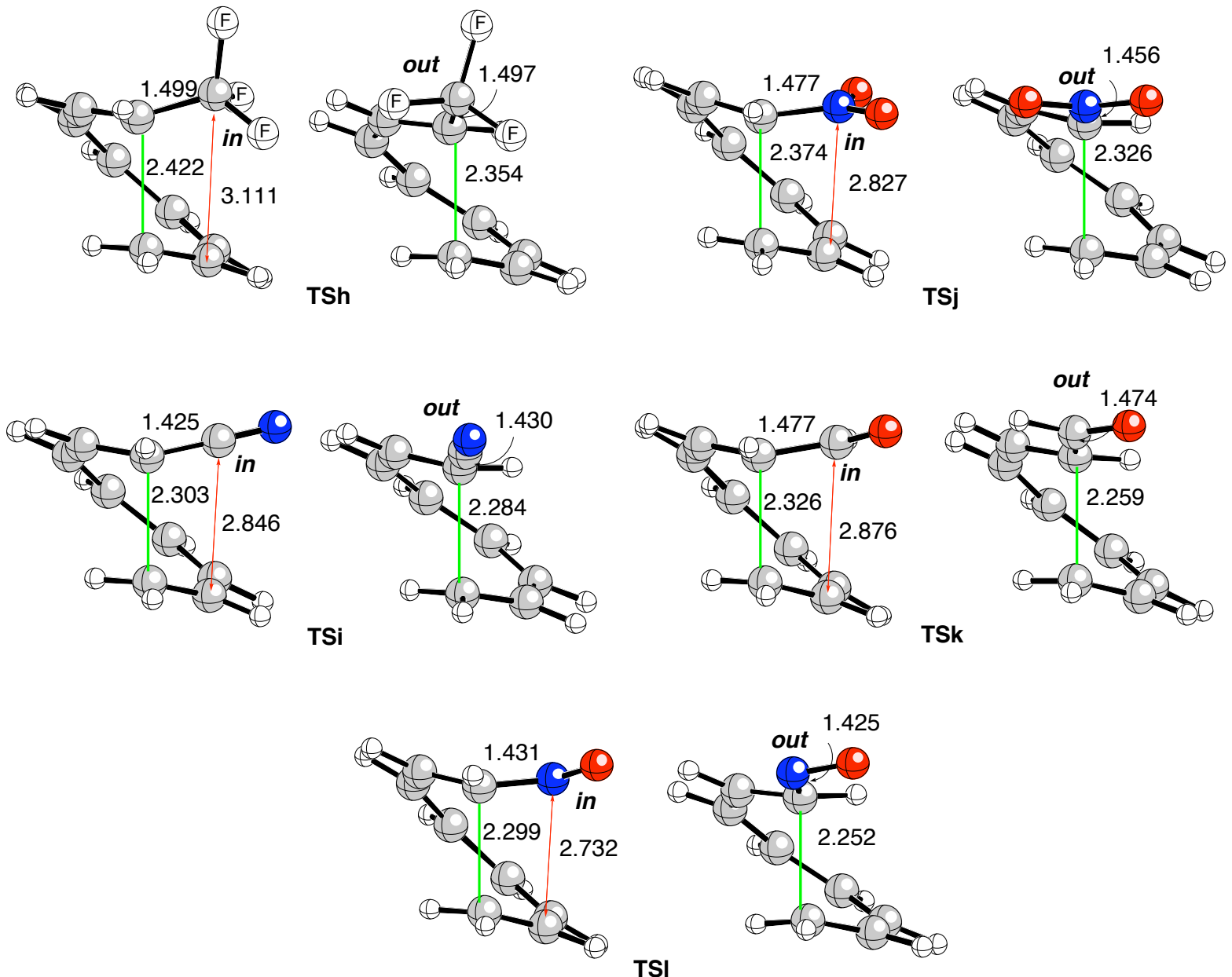

Figure S2. Chief geometric features (B3LYP/6-31G* level of theory) of transition structures in- and out-TSh-I (see Scheme 2). Bond distances are given in Å. Unless otherwise stated, white, gray, red and blue colors denote hydrogen, carbon, oxygen and nitrogen atoms, respectively. 